\title{
CRIRES spectroscopy and empirical line-by-line identification of FeH molecular absorption in an M dwarf ${ }^{\star}, \star \star$
}

\author{
S. Wende ${ }^{1}$, A. Reiners ${ }^{1}$, A. Seifahrt ${ }^{2}$, and P. F. Bernath ${ }^{3}$ \\ ${ }^{1}$ Institut für Astrophysik, Georg-August-Universität Göttingen, Friedrich-Hund-Platz 1, 37077, Germany \\ e-mail: sewende@astro.physik.uni-goettingen.de, Ansgar.Reiners@phys.uni-goettingen.de \\ 2 Physics Department Univ. of California, One Shields Avenue Davis, CA 95616, USA \\ e-mail: seifahrt@physics.ucdavis.edu \\ 3 Department of Chemistry, University of York, Heslington, York, YO10 5DD, UK \\ e-mail: pfb500@york.ac.uk
}

Received 16 June 2010 / Accepted 22 July 2010

\begin{abstract}
Molecular FeH provides a large number of sharp and isolated absorption lines that can be used to measure radial velocity, rotation, or magnetic field strength with high accuracy. Our aim is to provide an $\mathrm{FeH}$ atlas for M-type stars in the spectral region from $986 \mathrm{~nm}$ to $1077 \mathrm{~nm}$ (Wing-Ford band). To identify these lines in CRIRES spectra of the magnetically inactive, slowly rotating, M5.5 dwarf GJ1002, we calculated model spectra for the selected spectral region with theoretical FeH line data. In general this line list agrees with the observed data, but several individual lines differ significantly in position or in line strength. After identification of as many as possible $\mathrm{FeH}$ lines, we corrected the line data for position and line strength to provide an accurate atlas of $\mathrm{FeH}$ absorption lines for use in high precision spectroscopy of low mass stars. For all lines, we used a Voigt function to obtain their positions and equivalent widths. Identification with theoretical lines was done by hand. For confirmation of the identified lines, we used statistical methods, cross-correlation techniques, and line intensities. Eventually, we were able to identify FeH lines from the $(0,0),(1,0),(1,1),(2,1)$, $(2,2),(3,2)$, and $(4,3)$ vibrational bands in the observed spectra and correct the positions of the lines if necessary. The deviations between theoretical and observed positions follow a normal distribution approximately around zero. In order to empirically correct the line strength, we determined $T_{\text {eff }}$, instrumental broadening (rotational broadening) and a van der Waals enhancement factor for the $\mathrm{FeH}$ lines in GJ1002. We also give the scaling factors for the Einstein A values to correct the line strengths. With the identified lines, we derived rotational temperatures from the line intensities for GJ1002. We conclude that FeH lines can be used for a wide variety of applications in astrophysics. With the identified lines it will be possible for example to characterize magnetically sensitive or very temperature sensitive lines, which can be used to investigate M-type stars.
\end{abstract}

Key words. molecular data - line: identification - line: profiles - stars: late-type

\section{Introduction}

High-resolution spectroscopy of atomic or molecular lines is used to measure rotation, magnetic fields, and radial velocity variations. In solar-like stars, atomic lines are useful to measure these quantities because they are numerous, well isolated, and sufficiently narrow. In cooler stars, like M dwarfs, atomic lines are no longer useful because most of them become very weak, others become strongly pressure-broadened and they are usually overlapped by strong molecular bands. In these stars molecular lines are a valuable tool to measure the quantities mentioned above. However, molecular lines tend to cluster in dense bands, e.g. for $\mathrm{TiO}$ and $\mathrm{VO}$ in the visual spectral range. Only a few molecules provide absorption lines that are isolated and can be used for detailed spectroscopic analysis.

The FeH molecule provides a particularly large number of strong and well isolated lines in the $z$-band ( 990-1080 nm). It is the main opacity contributor in this region for late-type

\footnotetext{
* Data were taken at ESO Telescopes under the program 79.D0357(A).

$\star \star$ Full Table 4 and $\mathrm{FeH}$ atlas are only available in electronic form at the CDS via anonymous ftp to

cdsarc.u-strasbg.fr (130.79.128.5) or via

http://cdsweb.u-strasbg.fr/viz-bin/qcat?]/A+A/523/A58
}

dwarf stars, and can be used for high-precision spectroscopy. $\mathrm{FeH}$ provides numerous unblended lines that are sufficiently narrow to measure small broadening effects or variations in the line position.

Wing \& Ford (1969) first discovered the molecular band around $991 \mathrm{~nm}$ in the spectra of the cool dwarf Wolf 359. This band was also found in S-type stars (Wing 1972) and was identified as the $(0,0)$ vibrational band of the $\mathrm{FeH}$ molecule by Nordh et al. (1977). An extensive analysis was carried out by Phillips et al. (1987). They identified seven vibrational bands of the ${ }^{4} \Delta-{ }^{4} \Delta$ electronic transition of the $\mathrm{FeH}$ molecule and provided tables with molecular constants and quantum numbers. An important theoretical work, partly based on the previous one, was carried out by Dulick et al. (2003). They computed a line list for the $F^{4} \Delta-X^{4} \Delta$ electronic transition and provided extensive tables of molecular data with quantum numbers and line intensities.

$\mathrm{FeH}$ absorption bands were also detected in the $J$ - and $H$-band with medium resolution spectra (Cushing et al. 2003). In the $H$-band the $E^{4} \Pi-A^{4} \Pi$ electronic transition is visible (Hargreaves et al. 2010). That $\mathrm{FeH}$ can be used to determine effective temperatures was shown for example by Schiavon et al. (1997) or Wende et al. (2009), and its potential to measure magnetic field strengths was demonstrated by 
Reiners \& Basri (2006, 2007). Theoretical work on the magnetic sensitivity of FeH was published by Afram et al. (2007, 2008).

In this paper we use high-resolution spectra of GJ1002 to empirically verify the line list of Dulick et al. (2003) and identify $\mathrm{FeH}$ on a line-by-line basis in the region $989.8-1076.6 \mathrm{~nm}$. For this purpose we will use Voigt functions to determine the empirical positions and equivalent widths of the observed $\mathrm{FeH}$ lines. We identify the observed lines with theoretical ones by hand, and confirm this identification using statistical means, crosscorrelation techniques, and the line strength of the identified FeH lines. Furthermore, we correct theoretical Einstein A values to account for mismatches in line depth. We also derive rotational temperatures from the identified lines, and investigate under which circumstances they are close to the effective temperature of the star.

\section{Data}

\subsection{CRIRES spectra of GJ1002}

The observational data are CRIRES spectra of the inactive $M$ 5.5 dwarf GJ1002 (see Fig. 1). The M dwarf has an assumed effective temperature of $3150 \mathrm{~K}$ (from the spectral type), and it is a very slow rotator ( $v \sin i<3 \mathrm{~km} \mathrm{~s}^{-1}$, Reiners \& Basri 2007). There is also very low $H_{\alpha}$ and $\mathrm{X}$-ray activity from which we can assume that the magnetic field strength is relatively low. Owing to the weak magnetic field and slow rotation, GJ1002 is an ideal target for identification of molecular $\mathrm{FeH}$ lines. The lines are only slightly broadened by the different possible mechanisms in contrast to observations in sun spots where $\mathrm{FeH}$ lines are always influenced by a strong magnetic field.

CRIRES observations of GJ1002 were conducted in service mode during several nights in July 2007. The entrance slit width was set to $0.2^{\prime \prime}$, hence, the nominal resolving power was $R \sim$ 100000 . Four frames with an integration time of five minutes were taken in an ABBA-nod pattern for each of the nine wavelength settings covering the region between 986-1077 nm, leaving only one larger gap at $991.15-992.45 \mathrm{~nm}$ and two smaller gaps at $997.15-997.50 \mathrm{~nm}$ and $1057.15-1057.65 \mathrm{~nm}$.

Data reduction made use of the ESOREX pipeline for CRIRES. Science frames and flatfield frames were corrected for non-linearity and 1D spectra were extracted from the individual flatfielded and sky subtracted frames with an optimum extraction algorithm. The wavelength solution is based at first order on the Th-Ar calibration frames provided by ESO. Due to the slit curvature, spectra taken in B-nodding positions are shifted in wavelength with respect to spectra taken in A-nodding positions. This was corrected for by mapping all $1 \mathrm{D}$ spectra to the spectrum of the first A-nod position.

The individual CRIRES wavelength settings provide a considerable degree of spectral overlap and up to eight individual spectra were combined into one final spectrum at each wavelength. While merging the individual settings, small mismatches in the wavelength solutions as well as imperfections in the individual spectra (detector cosmetics, ghost contamination) were corrected. The final spectrum was normalized to a pseudo-continuum level of unity and finally shifted to match the McMath FTS spectrum of solar umbra (Wallace et al. 1998). The error in the wavelength calibration should be smaller than $0.75 \mathrm{~km} \mathrm{~s}^{-1}$.

We find the SNR at the continuum level of most parts of the final spectrum to exceed 200. The high signal-to-noise ratio and high spectral resolution of the CRIRES data allow us to identify FeH lines with high accuracy.

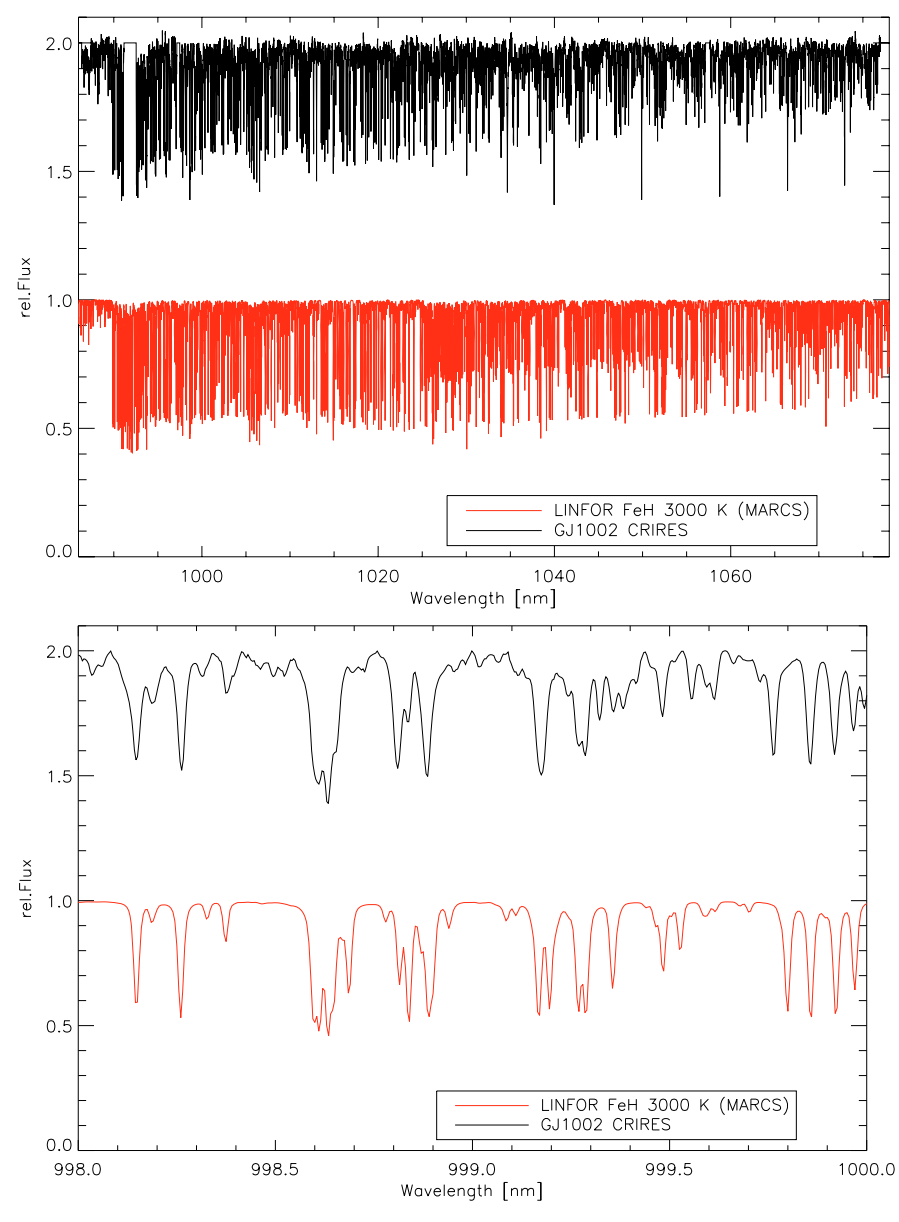

Fig. 1. top: FeH Wing-Ford band, observed (black) and computed (red). Bottom: a magnification of the spectrum above.

\subsection{Theoretical $\mathrm{FeH}$ molecular data and line synthesis}

The theoretical data which we use to identify the $\mathrm{FeH}$ lines is taken from Dulick et al. (2003). They provide tables of quantum numbers and energies ${ }^{1}$. In particular, they provide the vibrational assignment of the upper and lower states $v_{\mathrm{u}}$ and $v_{\mathrm{l}}$, respectively, the projection of the total orbital angular momentum on to the internuclear axis for the upper and lower state, $\Omega_{\mathrm{u}}$ and $\Omega_{1}$, and the rotational quantum number $J_{1}$ for the lower state. Furthermore the transition branch $(\mathrm{P}, \mathrm{Q}, \mathrm{R})$, the parity, the wavenumber in $\mathrm{cm}^{-1}$, the lower state energy of the transition $E_{1}$ and the Einstein A value are given.

From this information it is possible to estimate $\log _{10} g f$ values through (Bernath 2005)

$g_{1} f=\frac{\epsilon_{0} m_{\mathrm{e}} c^{3}}{2 \pi \mathrm{e}^{2} v^{2}} A g_{\mathrm{u}}$

with $g_{1}=2 J_{1}+1$ and $g_{\mathrm{u}}=2 J_{\mathrm{u}}+1$ as the lower and upper statistical weights of the transition and $v=c / \lambda$ as the transition frequency. All quantities are in SI units.

The van der Waals broadening is determined following Schweitzer et al. (1996), which is basically Unsöld's hydrogenic approximation. For the ionization energy needed in this approximation we used an empirically determined value of $6 \mathrm{eV}$, which was deduced from comparison with other diatomic molecules (Wende et al. 2009). Although this is not the theoretical value,

${ }^{1}$ See http://bernath. uwaterloo.ca/FeH 
which is slightly higher, we used this one because its influence on the van der Waals broadening is not significant.

The molecular partition function for $\mathrm{FeH} Q_{\mathrm{FeH}}$, which is needed for the concentration of $\mathrm{FeH}$, is computed after Sauval \& Tatum (1984) with molecular data taken from Tables 9 and 10 of Dulick et al. (2003). We give Eq. (2) in a polynomial expression of a fit to the partition function which is valid between $1000 \mathrm{~K}$ and $8000 \mathrm{~K}$.

$Q_{\mathrm{FeH}}=\sum_{i=0}^{4} a_{i} T^{i}$,

where $T$ is the temperature in $\mathrm{K}$ and

$\left(\begin{array}{l}a_{0} \\ a_{1} \\ a_{2} \\ a_{3} \\ a_{4}\end{array}\right)=\left(\begin{array}{r}-4.9795007 e+02 \\ 6.5460944 e-01 \\ 3.4171590 e-04 \\ 2.7602574 e-07 \\ 1.0462656 e-11\end{array}\right)$,

are the coefficients of the polynomial. For the creation of $\mathrm{FeH}$, i.e. the concentration that is governed by the Saha-Boltzmann equation, we assume $\mathrm{Fe}+\mathrm{H} \rightarrow \mathrm{FeH}$. With this partition function and the data from Dulick et al. (2003) we can use a simple description for the absorbance (described in Sect. 3.4 in this paper in more detail) to compute $\mathrm{FeH}$ spectra with a simple reversing layer model and separate the lines into vibrational bands in the observed wavelength region (see Fig. 2). From this figure we can expect that there will be two sequences of vibrational transitions, namely the sequence with $\Delta v=0$, which are the $(0,0),(1,1)$, and $(2,2)$ vibrational transitions, and the sequence with $\Delta v=1$, which are the $(1,0),(2,1),(3,2)$, and $(4,3)$ transitions. We note that this method does not take into account (among many other things) the atmospheric structure or the chemical composition of the star. It gives only a rough estimate of the relative strengths of the FeH bands.

The full synthetic line formation for the comparison and identification of the observed $\mathrm{FeH}$ lines is done with the line formation code SYNTH3 (Kochukhov 2007). This code is able to compute large spectral regions with all $\mathrm{FeH}$ lines taken from our line list (see Fig. 1). We used only lines with $\log _{10} g f>-7$ because we assume that other lines have no significant influence. By computing all the lines in a certain region at once, we accounted for blends, but we also computed all lines of the region individually to measure their equivalent width $W_{\lambda}$ with the LINFOR3D code (based on Baschek et al. 1966). For the input model atmospheres we used MARCS (Gustafsson et al. 2008) with solar composition (Grevesse et al. 2007). These models are well suited for these cool temperatures in low-mass stars, because they make use of up-to-date atomic and molecular data and reach to effective temperatures of $2500 \mathrm{~K}$. We used the plane-parallel LTE models where the convection is treated in the mixing-length approximation. In the computation of these model atmospheres, the microturbulence parameter was set to zero. However, in the computations of the actual spectra, we assumed microturbulence parameters according to the results of Wende et al. (2009). We do not expect any significant influence because they are on the order of a few hundred $\mathrm{m} \mathrm{s}^{-1}$. We neglect the broadening from macro-turbulent motion, which would be hardly visible in the observed spectra.

\section{Methods}

We began with the investigation of the observed spectrum, for which we determined the position of the spectral lines and decide whether a line feature is a blend or not. Then we measured

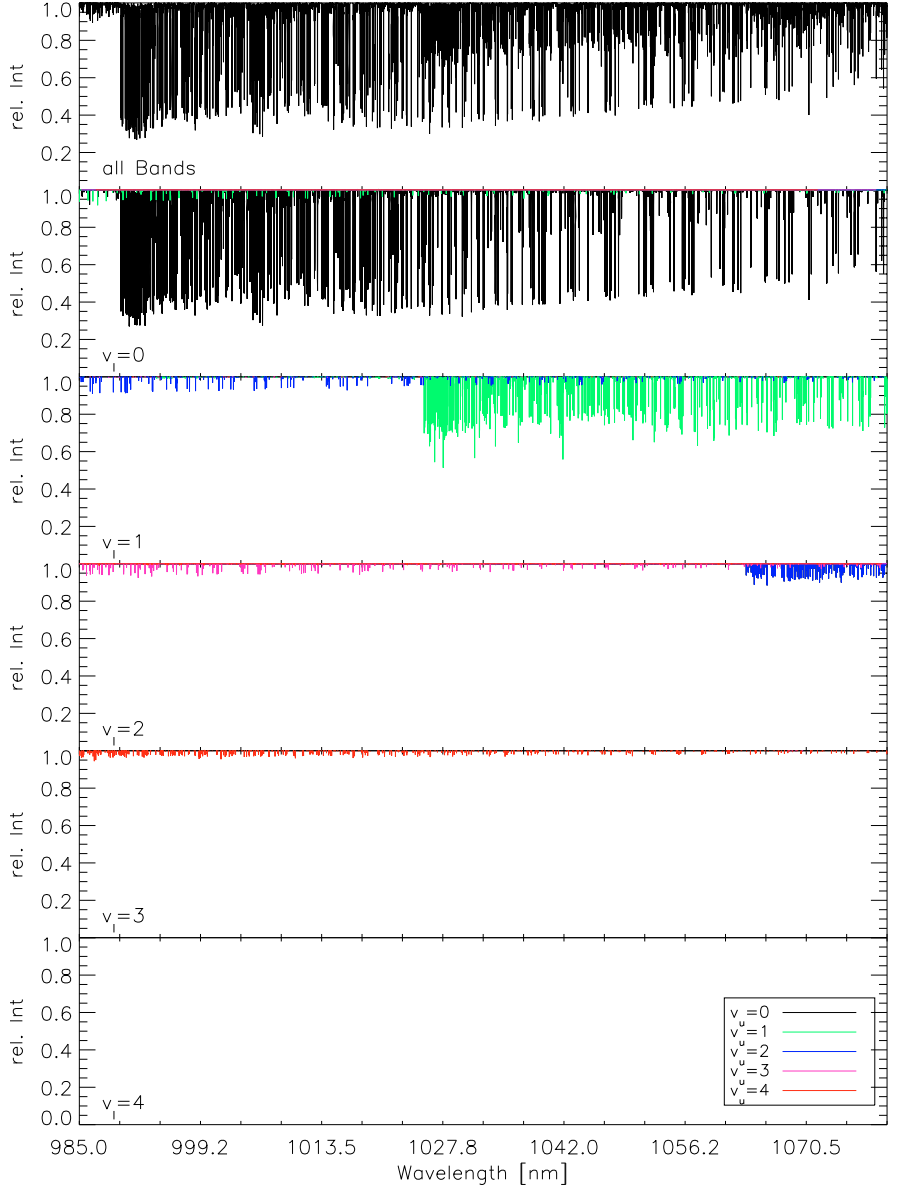

Fig. 2. FeH vibrational bands separated by their vibrational quantum numbers. See color version for more details.

the equivalent width $W_{\lambda}$ of the lines with a Voigt-fit procedure described below.

We compared the line positions found in the CRIRES data to theoretical ones and identified them with FeH lines. In order to confirm an identification, we used statistical means: (i) the method of coincidence, and a cross-correlation technique producing coincidence curves; and (ii) a method which takes the intensity into account. In this latter method we will compare the theoretical line strength $S$ (Hönl-London factor) with the observed $W_{\lambda}$ following Schadee (1964). We also use a description for the absorbance of spectral lines to correct theoretical line intensities given in terms of the Einstein A values. For this we will compare the observed and computed spectra with each other and obtain a scaling factor for the Einstein A values. The final result is a corrected line list that reproduces the observed stellar spectrum as well as the line positions in sunspot spectra from Wallace et al. (1999). The line intensities are hard to confirm in the solar case, because many $\mathrm{FeH}$ lines are strongly split by magnetic fields.

\subsection{Voigt fit}

In order to measure $W_{\lambda}$ in the observed spectra we used a "multiVoigt fit" procedure (based on IDL curvefit function). This is defined as

$F=\sum{ }_{i=0}^{N} A_{i} \frac{H\left(u_{i}, a_{i}\right)}{\max \left(H\left(u_{i}, a_{i}\right)\right)}$, 


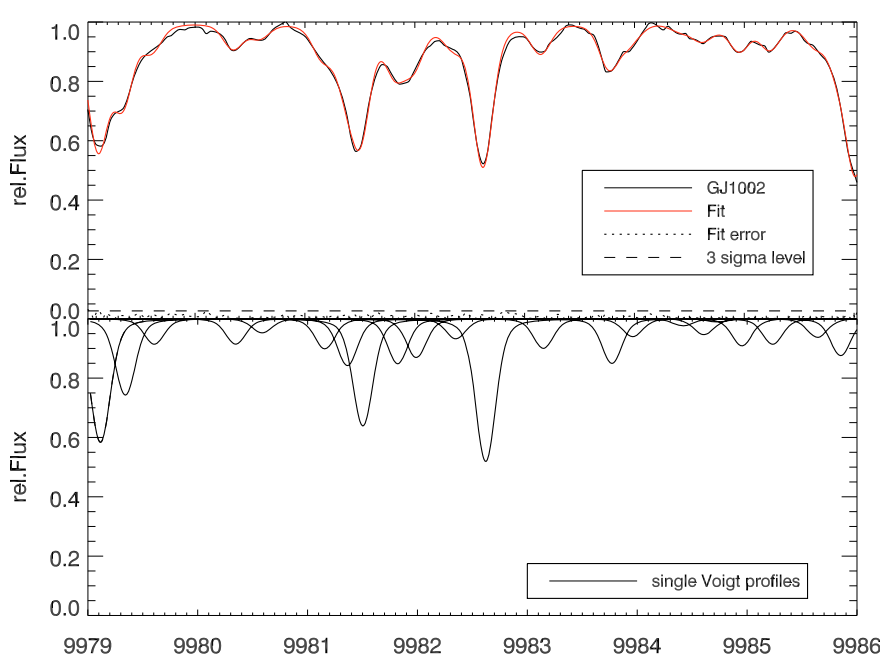

Fig. 3. Upper panel: part of the observed spectrum of GJ1002 with multiple Voigt fit. Lower panel: the single Voigt functions which were returned by the fit.

where $\mathrm{A}$ is the amplitude describing the depth of the line,

$u=\frac{\lambda-\lambda_{0}}{\sigma}$,

with $\sigma$ as Gaussian (or Doppler) width,

$$
a=\frac{\gamma}{4 \pi} \frac{\lambda_{0}^{2}}{c} \frac{1}{\sigma}
$$

which will be called the Voigt constant throughout in this paper, $\gamma$ is the radiation damping constant, and $H(u, a)$ is the Hjerting function (Gray 2008, and references therein). We successively fitted the whole spectrum within bins of $7 \AA$. The first and the last $1 \AA$ were cut off after the fit to avoid boundary effects, and we used only line profiles whose centers are inside the $5 \AA$ bin. For the Voigt profiles inside a bin, we assumed a constant $a$ value for the Lorentz part and a fixed Gaussian width, but we allow changes in these two parameters from one wavelength bin to another because the $\mathrm{FeH}$ lines tend to become narrower towards longer wavelengths. This is probably because we used $a$ and $\sigma$ only as fit parameters, and the FeH lines start to saturate at the band head, but become weaker towards longer wavelengths. Hence, the width of the line profile, which is a combination of $a$ and $\sigma$, decreases with decreasing saturation and consequently both parameters decrease. The wavelength dependence of the Doppler width affects the width of the lines as well, but it is negligible and goes in opposite direction (for example, $\sigma=0.1 \AA$ at $\lambda=10000 \AA$ would change to $\sigma=0.105 \AA$ at $\lambda=10500 \AA$ ).

With this fit procedure, we obtained the parameters (position, depth (amplitude), Voigt constant, and $\sigma$ ), needed for the individual Voigt line-profiles to fit the observed spectrum. In Fig. 3 we show an example of how the fit (red) reduced the observed spectrum (black) into single-line profiles (bottom panel).

The convergence criterion is the minimization of the residual flux between observation and fit $(O-C)$. The iteration is stopped if the maximal error of the fit is lower than three times the standard deviation of the error or if the standard deviation of the error does not change significantly between two iterations. From these Voigt profiles, $W_{\lambda}$ can easily be computed by integrating over the single-line profiles. The fit is also able to find and separate possible blends. For this, we assume that the blended components differ in position by at least $0.1 \AA$.
The measured $W_{\lambda}$ will be assigned to the associated theoretical lines. This means that if an observed line can be identified with exactly one theoretical line, $W_{\lambda}$ is fully assigned to this one theoretical line. However, in most cases the theory predicts more than one line for an observed line position because of numerous overlapping vibrational bands in the same wavelength region and from many closely-spaced line pairs, which only differ in parity. Then $W_{\lambda}$ will be distributed to all predicted theoretical lines at this position. This is done with the ratio $R_{i}$ of theoretical equivalent widths ${ }^{\text {theo }} W_{\lambda}^{i}$, which is obtained from the individual theoretical line profiles:

$$
R_{i}=\frac{{ }^{\text {theo }} W_{\lambda}^{i}}{\sum_{i \in B}{ }^{\text {theo }} W_{\lambda}^{i}},
$$

with $B$ as the set of blended components. There is of course the chance that $\mathrm{FeH}$ is not the only contributor to the observed blend. To avoid this uncertainty, we additionally used the line intensities in a method described below.

\subsection{Method of coincidence}

To confirm the identification of a molecular band, or a sub-band, we used the "method of coincidence", which was first introduced by Russell \& Bowen (1929). It gives the number of lines $C$ in a spectral range that will be found by chance if one uses a set of randomly generated line-positions and compares them with observed ones. Fundamental probability calculations lead to

$C=M[1-\exp (-2 x w)]$,

where $M$ is the number of lines in a particular region, $x$ is the tolerated deviation in position, and $w$ is the line density (average number of spectral lines in the investigated region). This means that $C / M=p_{\text {random }}$ gives the probability of finding with a randomly chosen line position a random coincidence. If one identifies $N$ out of $M$ lines in the observations, then the probability of finding an identification is $N / M=p_{\text {identified }}$ The ratio $N / C$ describes how likely it is to find a line by random coincidence per identified line. Hence, the number of actually identified lines $N$ should exceed the number of $C$ of purely random coincidences. If this is the case, then one can assume that the lines are probably identified in the observed spectra.

\subsection{Theoretical line strength}

For the identification of molecular lines it is also useful to take the intensities of the lines into account. To compare the theoretical line strength $S$ with the observed equivalent width $W_{\lambda}$, we follow Schadee (1964). For weak lines (mildly saturated) $W_{\lambda}$ is proportional to the wavelength $\lambda_{0}$, the oscillator strength $f$, and $N_{i}$, the number of absorbers (Gray 2008):

$W_{\lambda} \propto \lambda_{0}^{2} f N_{i}$,

and

$f \propto \frac{g_{\mathrm{u}}}{g_{\mathrm{l}}} \lambda_{0}^{2} A$,

with $N_{i}$ as the number of absorbers given by the Boltzmann distribution

$N_{i} \propto g_{1} \mathrm{e}^{\left(\frac{-h c}{k T} E_{0}\right)}$,

where $E_{0}$ is the lower-state energy level in $\mathrm{cm}^{-1}$. To derive the final form, we use Eqs. (10) and (11) together with Eq. (9) and 


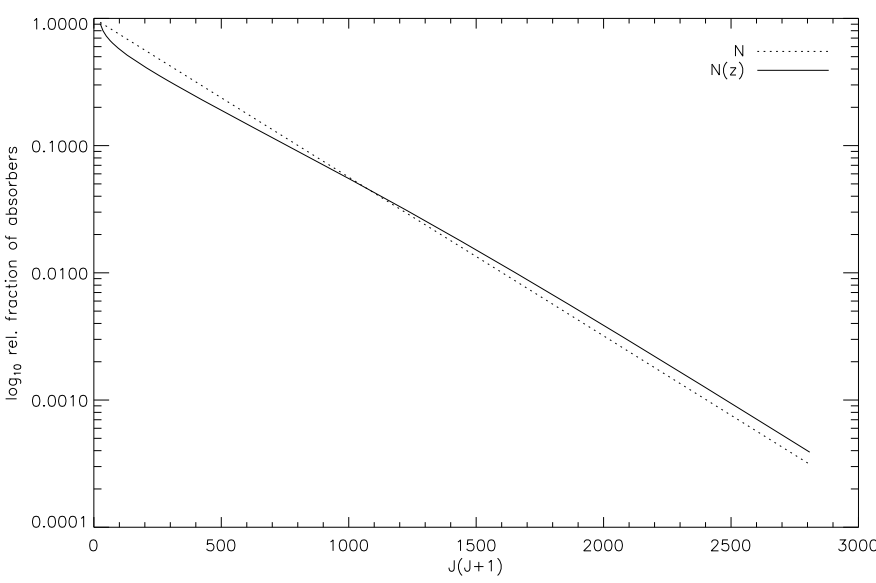

Fig. 4. Relative number of absorbers with (dashed line), and without (solid line) constant number of $\mathrm{FeH}$ molecules over different atmospheric layers.

the expression for the Einstein A value

$A=\frac{A_{v_{\mathrm{u}}, v_{1}} S}{g_{\mathrm{u}}}$.

Here $A_{v_{u}, v_{1}}$ is the Einstein $A$ for a specific vibrational transition and constant for the vibrational band. The resulting equation is

$\log _{10} \frac{W_{\lambda}}{S \lambda_{0}^{4}}=C-\frac{h c}{k T} E_{0} \log _{10} e$,

which is the relation from Schadee (1964) with $E_{0}$ replacing $B J_{1}\left(J_{1}+1\right)$. Possible stimulated emission can be neglected for our analysis because there is only a small population in the excited state. If we plot $\log _{10} W_{\lambda} / S \lambda_{0}^{4}$ against $E_{0}$, a straight line should be found, which then suggests the correct identification of the lines in the FeH molecular band. Equation (13) accounts for different branches as well as for different $\Omega$. This is because $S$ on the left side of Eq. (13) is computed for all branches (P, Q, R) and $\Omega$ values.

The $S$ values that we will use in the analysis are computed for the intermediate Hund's case and are determined from the Einstein $A$ values given by Dulick et al. (2003).

We point out that we implicitly assumed in Eq. (11) that the total number of $\mathrm{FeH}$ molecules $N$ is constant for all lines. This assumption is only valid if we consider a small isothermal atmospheric layer. However, because a spectral line forms over several layers, this assumption is not exactly valid. If we furthermore consider a set of lines with a wide range in $g f$ and $E_{0}$ values, we expect that these lines form at different heights. Thus, the number of $\mathrm{FeH}$ molecules is not constant anymore: strong spectral lines are assumed to be formed in higher atmospheric layers, and weak lines in deeper layers. Hence, deviations from a straight line in the $\left[\log _{10} W_{\lambda} / S, E_{0}\right]$ diagram for lines with very low and very high $J$ are expected. If one assumes more $\mathrm{FeH}$ molecules in deeper layers, larger equivalent widths for these lines can be expected owing to higher density. For a qualitative description, we assume the total number of $\mathrm{FeH}$ molecules to be inversely proportional to the equivalent width, which reflects the heights of formation for weak and strong lines.

$N(z)=N \alpha W_{\lambda}^{\beta}$.

$\alpha$ and $\beta$ are free parameters and here chosen as 1.25 and -0.25 , respectively. We plot the right hand side of Eq. (13) for the constant and variable molecule number in Fig. 4. Still, the situation is much more complicated, and we use synthetic line formation to investigate this behavior in the results section.

Following Schadee (1964), we can now use the $\left[\log _{10} W_{\lambda} / S, E_{0}\right]$ diagram to classify the identified $\mathrm{FeH}$ lines into one of the following classes:

1. $\mathrm{P}$ - the line is present, and its $W_{\lambda}$ agrees well with the straight line of the diagram.

2. $\mathrm{Pb}-$ the line is present, but its $W_{\lambda}$ is too large, i.e. the $\log _{10} W_{\lambda} / S$ value lies above the straight line of the diagram. This could imply that the line is blended by another element (or that its computed line strength is too weak).

3. $\mathrm{R}-$ the line strength is presumably reduced by perturbations. That means that the computed line strength is too great and the data point lies below the line.

4. Q - we identified the line, but we can not verify its identification, because we only investigate lines with $\Delta \Omega=0$ in this plot.

Eventually, it should be possible to derive the excitation temperature for the rotational transitions $T$ in Eq. (13) from the slope of a linear fit in the $\left[\log _{10} W_{\lambda} / S, E_{0}\right]$ diagram. Yet one has to be careful. Wöhl (1970) reports that the obtained rotational temperature from this method crucially relies on the data points which are included in the linear fit and also on the degree of accuracy in measuring $W_{\lambda}$. We also experienced the same difficulties.

\subsection{Line strength correction}

In some cases $W_{\lambda}$ and line depths of the observed FeH lines do not match the computed ones from the line list. In general, we observe that the differences increase towards longer wavelengths and computed lines become stronger than observed ones. The strength of the lines are mainly determined by the lower state energies $E_{0}$ and the Einstein A values. We will correct only the Einstein A values, because a set of high-resolution spectra in the $z$-band for different temperatures would be required to correct $E_{0}$. To correct the Einstein A value, we use the formula from Bernath (2005) for the absorbance in a modified form where the Einstein A values enter the expression

$-\ln \left(\frac{I}{I_{0}}\right)_{x}=\frac{\left(2 J_{\mathrm{u}}+1\right) A_{x}}{8 \pi \bar{v}^{2} q} \mathrm{e}^{-E_{0}^{x} / k T}\left(1-\mathrm{e}^{-h v / k T}\right) G N l$,

where $\bar{v}$ is the frequency of a molecular in $\mathrm{cm}^{-1}, N$ is the number of molecular absorbers per cubic $\mathrm{cm}$ in the energy state (population density), $q$ is the partition function, $l$ is the length, e.g. for an atmospheric layer, and $G$ is a line profile function, e.g. a Voigt function. If we compare the observed spectra with the computed one (produced with the SYNTH3 code) and assume that both atmospheres have the same structure, then $A_{x}$ and $E_{0}^{x}$ are the only variables that account for differences in the spectra ( $x$ stands for either the computed or observed spectra). We also assume $E_{0}^{x}$ as constant and write

$\frac{-\ln \left(\frac{I}{I_{0}}\right)_{\mathrm{obs}}}{-\ln \left(\frac{I}{I_{0}}\right)_{\mathrm{comp}}}=\frac{A_{\mathrm{obs}}}{A_{\mathrm{comp}}}$

This shows that the correction for the Einstein A value is merely a linear scaling with the ratio of the intensities

$A_{\mathrm{obs}}=\frac{-\ln \left(\frac{I}{I_{0}}\right)_{\mathrm{obs}}}{-\ln \left(\frac{I}{I_{0}}\right)_{\mathrm{comp}}} A_{\mathrm{comp}}=s A_{\mathrm{comp}}$. 


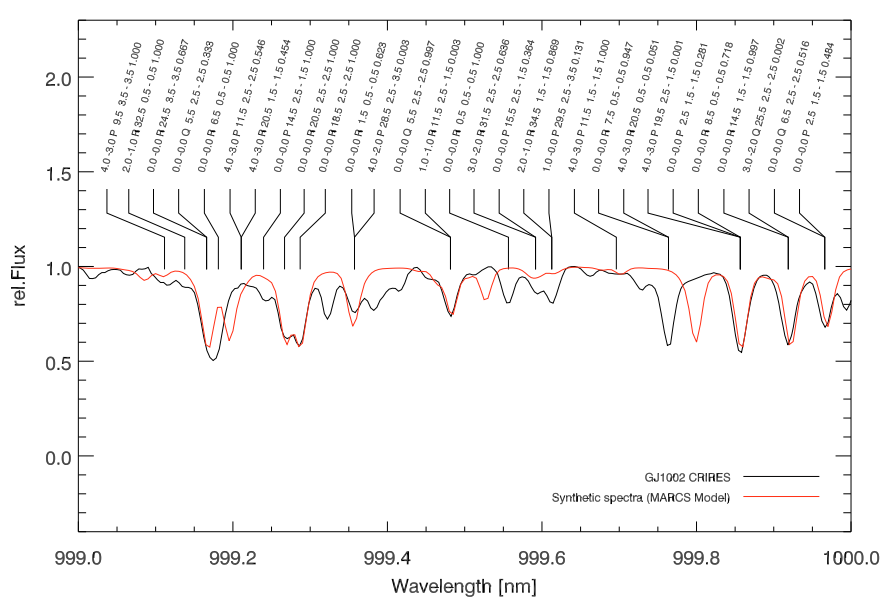

Fig. 5. Observed spectrum of GJ1002 (black) and computed one (red) labeled with quantum numbers.

We introduced the scaling factor $s$ for the ratio of both intensities. If we use that $A=A_{v_{\mathrm{u}}, \nu_{1}} S /\left(2 J_{\mathrm{u}}+1\right)$ (Dulick et al. 2003), then

$s=\frac{A_{v_{u}, v_{1}}^{\mathrm{obs}}}{A_{v_{\mathrm{u}}, v_{1}}^{\mathrm{comp}}} \frac{S_{\mathrm{obs}}}{S_{\mathrm{comp}}}$,

where $S$ is the Hönl-London factor. If the theoretical $S$ values are wrong, we could perhaps detect it using Eq. (13) or perhaps in a possible dependence of the scaling factors on $J_{1}$.

\section{Results}

\subsection{Atomic line identification and unidentified lines}

Because atomic lines are present in the wavelength region of the observed spectra of GJ1002, we use the VALD ${ }^{2}$ (Kupka et al. 1999; Piskunov et al. 1995) database to include the available atomic line data in our calculations. We give a list of atomic lines present at $T_{\text {eff }}=3100 \mathrm{~K}$ in Table 1 . We included lines deeper than $2 \%$ below the continuum in the computed spectra. We did not try to correct the atomic line positions or line strengths and took the data as provided by VALD.

After we identified the atomic lines and $\mathrm{FeH}$ lines, there were still unidentified lines that seemed to belong to neither FeH nor to a known atomic line. We give a list of these lines for which the line depth is deeper than $10 \%$ below the continuum (Table 2). Our opinion is that most of these lines belong to $\mathrm{FeH}$, but we were unable to identify them with confidence.

\subsection{FeH line identification}

We used the results from the Voigt fit to assign the individual Voigt profiles from the observed lines to the individual theoretical FeH lines. We did this by hand, and defined a line as identified if the position of the observed line agreed within $0.1 \AA$ to the theoretical predicted position. If the offset is larger than $0.1 \AA$, we identified a line feature if the characteristic shape was similar in the observations and computations (e.g. the line at $999.55 \mathrm{~nm}$ or $999.8 \mathrm{~nm}$ in Fig. 5). In Fig. 5, and also in the full FeH atlas ${ }^{3}$, we labeled all identified lines with their quantum numbers, i.e.

\footnotetext{
2 http://vald.astro.univie.ac.at

3 The $\mathrm{FeH}$ atlas is only available at the CDS.
}

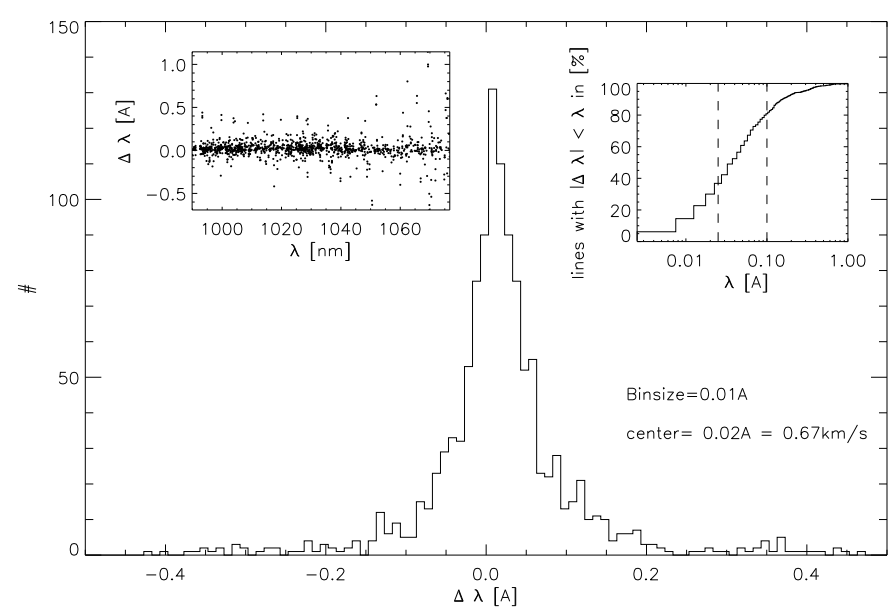

Fig. 6. Histogram of the residuals between computed line positions and observed ones. In the upper left corner, the residuals are plotted against wavelength. In the upper right corner, the fraction of lines with residuals lower than a certain value are plotted. The left dashed line represents the accuracy of the wavelength solution which is $0.025 \AA$, the right one the detection boundary of $0.1 \AA$.

vibrational assignment $\left(v_{\mathrm{u}}, v_{1}\right)$, branch $(\mathrm{P}, \mathrm{Q}, \mathrm{R})$, lower-state rotational quantum number $J, \Omega$, and in the case of blends, with their contribution to the blend. We did this for the observed wavelength region, and the complete plot is available in the online material of this paper. In Fig. 6 we show a histogram of the residuals between the computed line positions and the observed ones. There is no obvious systematic behavior of the scatter with wavelength (see inset in Fig. 6). The scatter follows a normal distribution centered at $\Delta \lambda=0.02 \AA$ corresponding to $0.67 \mathrm{~km} \mathrm{~s}^{-1}$. This mean value is beyond our spectral resolution, and in general all residuals smaller than $0.75 \mathrm{~km} \mathrm{~s}^{-1}(\sim 0.025 \AA)$ are not significant because they are also smaller than the accuracy of the wavelength calibration. From the investigation of the line positions, we saw that the fraction of lines for which the residuals are smaller than a certain range are distributed as shown in the right inlay of Fig. 6 . This plot shows that $\sim 80 \%$ of the line features could be identified by their positions, which do not deviate by more than $0.1 \AA$ from the predictions. Where the residuals are larger than $0.025 \AA$ the uncertainties in the molecular constants which were used to compute the $\mathrm{FeH}$ line list are responsible for these deviations.

For our analysis we used only lines with $W_{\lambda}>2 \mathrm{~m} \AA$, which approximately describes a line with $2 \%$ relative flux and a $F W H M$ of $0.1 \AA$. We ignored lines with smaller contributions because their intensities are similar to the noise level. However, only 167 lines out of 1359 have equivalent widths less than $2 \mathrm{m \AA}$.

\subsubsection{Vibrational bands}

From Fig. 2 we expect that the dominant vibrational bands are $(0,0)$ and $(1,1)$ from the $\Delta v=0$ sequence, and $(3,2)$ and $(4,3)$ from the $\Delta v=1$ sequence. In Fig. 7 we present a histogram with the number of identified lines for each vibrational band which shows that the expected vibrational bands are present. We also show the number of possible lines from theory with $W_{\lambda}>2 \mathrm{~m} \AA$ in the observed wavelength region. This number is based on lineby-line computation of $W_{\lambda}$. In this histogram the bars in the 
Table 1. Identified atomic lines.

\begin{tabular}{|c|c|c|c|c|c|c|c|c|c|c|c|}
\hline Ion & Position $[\AA]$ & $E_{1}$ & $\log g f$ & Ion & Position $[\AA]$ & $E_{1}$ & $\log g f$ & Ion & Position $[\AA]$ & $E_{1}$ & $\log g f$ \\
\hline "Cr1" & 9903.6226 & 2.987 & -2.131 & "Fe1" & 10116.787 & 2.759 & -3.705 & "Ti1" & 10399.651 & 0.848 & -1.623 \\
\hline "Ti1" & 9930.0728 & 1.879 & -1.580 & "Ti1" & 10123.668 & 2.175 & -1.722 & "Cr1" & 10419.476 & 3.013 & -1.806 \\
\hline "Ti1" & 9944.1036 & 2.160 & -1.821 & "Ti1" & 10148.300 & 3.148 & -0.910 & "Fe1" & 10425.885 & 2.692 & -3.627 \\
\hline "Ti1" & 9951.7317 & 2.154 & -1.778 & "Fe1" & 10148.342 & 4.796 & -0.177 & “Ti1” & 10462.915 & 2.256 & -2.054 \\
\hline "Cr1" & 9951.7997 & 3.556 & -1.129 & "Fe1" & 10157.947 & 2.176 & -4.225 & "Fe1" & 10472.522 & 3.884 & -1.187 \\
\hline "Ti1" & 9967.4679 & 1.053 & -4.108 & "Fe1" & 10170.256 & 2.198 & -4.114 & "Cr1" & 10489.119 & 3.011 & -0.972 \\
\hline "Ti1" & 10000.700 & 1.873 & -1.840 & "Ti1" & 10173.274 & 1.443 & -3.465 & “Ti1" & 8.990 & 0.836 & -1.739 \\
\hline "Ti1" & 10005.828 & 2.160 & -1.124 & "Fe1" & 10197.900 & 2.728 & -3.589 & "Cr1" & .887 & 3.013 & -1.558 \\
\hline "Ti1" & 10008.403 & 1.067 & -3.626 & "Ca1" & 10201.981 & 4.680 & -0.369 & "Fe1" & 121 & 3.929 & -1.482 \\
\hline "Ti1" & 10014.490 & 2.154 & -1.284 & "Ca1" & 10205.743 & 4.681 & -0.199 & "Cr1" & 10552.983 & 3.011 & -1.976 \\
\hline "Sc1" & 10027.787 & 1.865 & -1.286 & "Ca1" & 10205.803 & 4.681 & -1.102 & “Ti1" & 10554.648 & 1.887 & -2.607 \\
\hline "Ti1" & 10037.242 & 1.460 & -2.227 & "Ca1" & 10211.457 & 4.681 & -0.039 & "Fe1" & 10580.037 & 3.301 & -3.137 \\
\hline "Ti1" & 10051.581 & 1.443 & -2.205 & "Ca1" & 10211.561 & 4.681 & -1.102 & “Ti1" & 10587.533 & 0.826 & -1.866 \\
\hline "Sc1" & 10060.261 & 1.851 & -1.479 & "Fe1" & 10221.204 & 3.071 & -2.760 & “Ti1" & 10610.624 & 0.848 & -2.761 \\
\hline "Fe1" & 10060.397 & 5.033 & -1.231 & "Fe1" & 10268.031 & 2.223 & -4.533 & "Fe1" & 10619.630 & 3.267 & -3.128 \\
\hline "Ti1" & 10060.485 & 2.175 & -0.894 & "Ca1" & 10291.397 & 4.624 & -0.265 & "Cr1" & 10650.558 & 3.011 & -1.613 \\
\hline "Ti1" & 10062.662 & 1.430 & -2.351 & "Fe1" & 10343.720 & 2.198 & -3.574 & "Ti1" & 10664.544 & 0.818 & -2.007 \\
\hline "Fe1" & 10067.804 & 4.835 & -0.288 & "Ca1" & 10346.655 & 2.933 & -0.408 & "Cr1" & 10670.437 & 3.013 & -1.489 \\
\hline "Ti1" & 10069.273 & 2.160 & -1.750 & "Fe1" & 10350.802 & 5.393 & -0.548 & "Cr1" & 10675.063 & 3.013 & -1.374 \\
\hline "Ti1" & 10077.885 & 1.067 & -4.065 & "Fe1" & 10381.844 & 2.223 & -4.145 & “Ti1" & 10679.972 & 0.836 & -2.592 \\
\hline "Cr1" & 10083.115 & 3.556 & -1.307 & “Ti1" & 10393.591 & 1.502 & -2.595 & "Fe1" & 10728.124 & 3.640 & -2.763 \\
\hline "Fe1" & 10084.158 & 2.424 & -4.544 & "Cr1" & 10394.793 & 3.010 & -2.006 & “Ti1" & 10729.329 & 0.813 & -2.156 \\
\hline "Cr1" & 10114.770 & 3.013 & -2.073 & "Fe1" & 10398.645 & 2.176 & -3.390 & "Ca1" & 10729.654 & 4.430 & -1.841 \\
\hline
\end{tabular}

Table 2. List of unidentified lines deeper then 0.9.

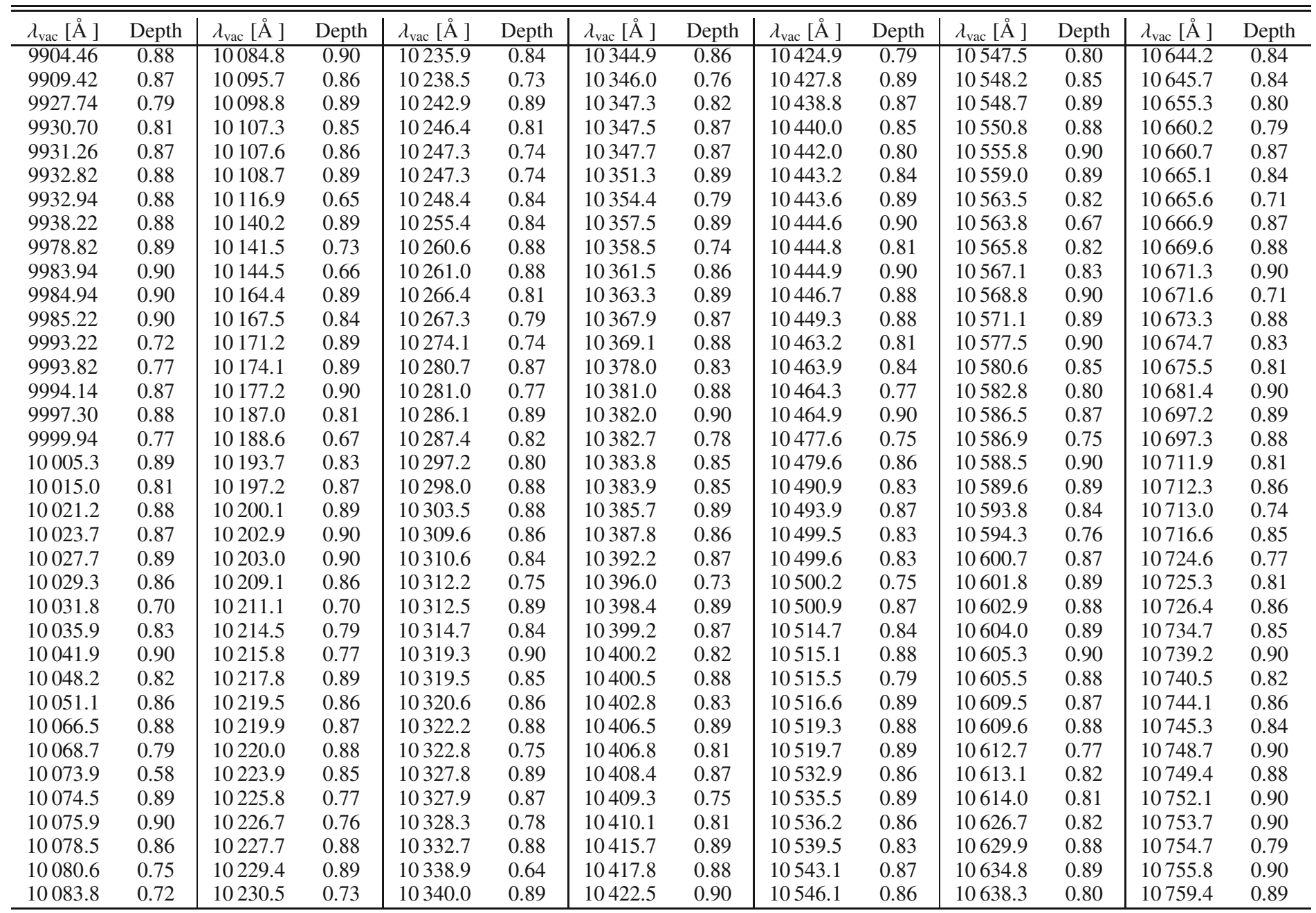




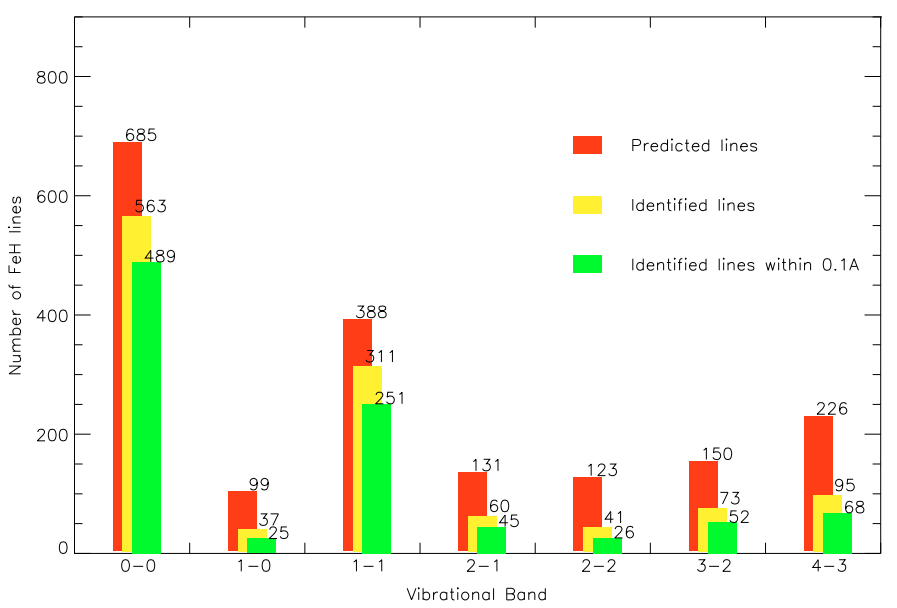

Fig. 7. Histogram of predicted and identified FeH lines in the GJ1002 spectrum.

Table 3. Results from the coincidence method for lines with $W_{\lambda}>$ $2 \mathrm{~m} \AA$.

\begin{tabular}{ccccc}
\hline \hline Band & Wavelength [̊] & $C$ & $w[\# / \AA]$ & $N(M)$ \\
\hline$(0,0)$ & $9900-10762$ & 360 & 3.73 & $489(658)$ \\
$(1,0)$ & $9901-10759$ & 52 & 3.74 & $25(99)$ \\
$(1,1)$ & $9941-10759$ & 208 & 3.71 & $251(388)$ \\
$(2,1)$ & $9931-10706$ & 68 & 3.76 & $45(131)$ \\
$(2,2)$ & $10480-10764$ & 65 & 3.81 & $26(123)$ \\
$(3,2)$ & $9910-10764$ & 79 & 3.78 & $52(150)$ \\
$(4,3)$ & $9905-10673$ & 119 & 3.75 & $68(226)$ \\
\hline
\end{tabular}

foreground take into account only the lines identified by their positions.

\subsubsection{Coincidence-by-chance method}

For the lines with residuals smaller than $0.1 \AA$ we computed the coincidence-by-chance factor $C$ from Eq. (8). In Table 3 the values of $C, w, N$, and $M$ are given for all identified bands. For the $(0,0)$ and $(1,1)$ transition, the number of identified lines $N$ exceeds the number of coincidences by chance $C$, which is a clear indication that these bands are present in the investigated region. For the other bands the situation is not as clear and we will confirm them with more investigations.

\subsubsection{Cross-correlation method}

We used cross-correlation techniques to investigate the agreement between the theoretical line list and the observed spectra. As a reference and a test, we cross-correlated a computed spectrum from this theoretical line list, which is broadened by an instrumental profile with a resolving power of 70000 , with the original line list (e.g. Fawzy 1995; Fawzy et al. 1998). To be specific, we varied the theoretical positions with steps of $\Delta \sigma=0.0125 \AA$ in a range of $0.375 \AA$ and measured the relative intensity at the different positions weighted with $W_{\lambda}$ of the line and integrate over all lines. We then normalized the results with the number of lines in the line list. We did this for all lines in the vibrational bands that are found in the M-dwarf spectra (see Fig. 8). If a vibrational band is present, a peak around zero appears above the noise produced by random coincidences with other lines.
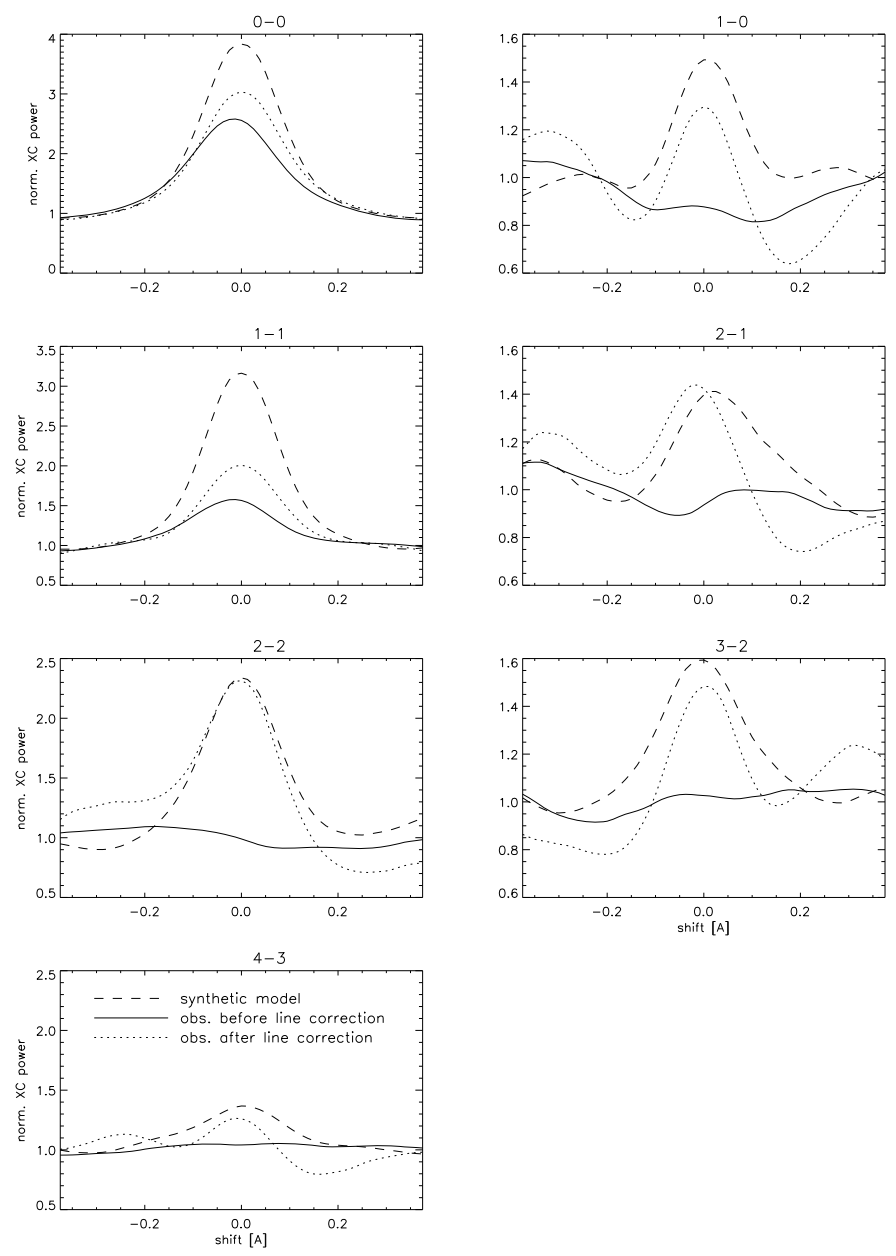

Fig. 8. Cross-correlation curves for different vibrational bands.

We produced three different curves. For the first one, we used all possible lines from the original line list for comparison with the observed stellar spectrum (solid line in Fig. 8). For the second curve we computed a reference curve by cross-correlating the original line list with a synthetic spectrum computed from it (dashed line in Fig. 8). This case produces the maximum possible correlation. A third curve is produced with the corrected line list containing only identified $\mathrm{FeH}$ lines which we compared with the observed stellar spectrum (dotted line in Fig. 8).

As expected, for the synthetic spectrum with the theoretical line list (dashed line), all bands show clear peaks above the noise. For the observed spectrum (solid line) and original line list, the $(0,0)$ and $(1,1)$ vibrational bands show clear peaks above the noise, which agrees with the coincidences by chance values in Table 3. After improving the FeH line list (dotted line), all bands show peaks above the noise similar to the peaks obtained in the reference case from cross-correlating the theoretical line list with the computed spectra. The original theoretical line positions for the $(1,0),(2,1),(2,2),(3,2)$, and $(4,3)$ bands are not accurate enough to show significant peaks in the cross correlation. To confirm our identifications, we also used the line strength in the next section.

\subsubsection{Line intensity method}

We used the method described in Sect. 3 to test if the tentatively identified observed $\mathrm{FeH}$ lines can be identified with the theoretical ones. We plot $\log _{10} W_{\lambda} / S \lambda_{0}^{4}$ against $E_{0}$ (see Eq. (13)) for the 
S. Wende et al.: CRIRES spectroscopy and empirical line-by-line identification of FeH molecular absorption in an M dwarf
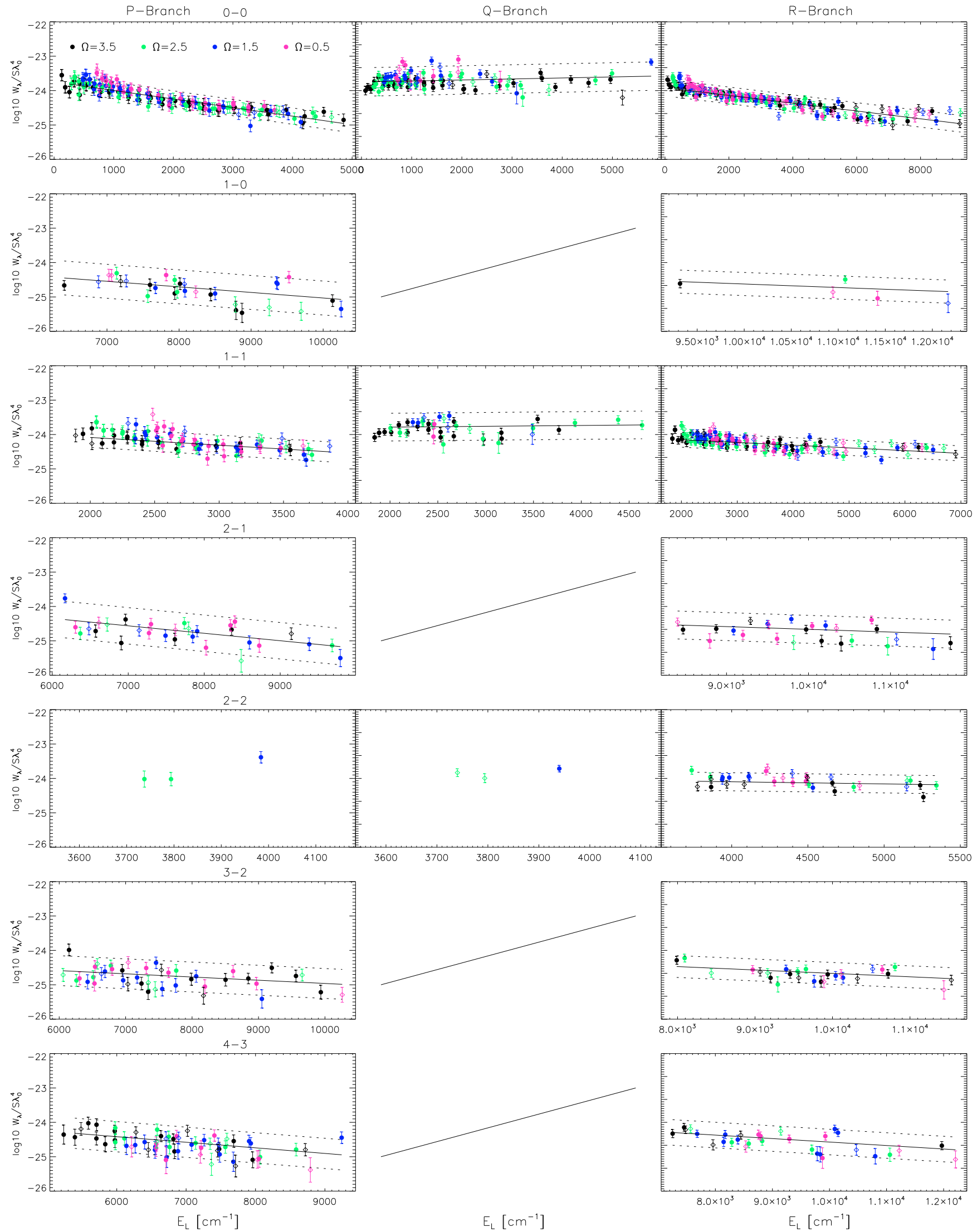

Fig. 9. Logarithm of observed equivalent width and theoretical line strength against lower level energies. In each plot, a linear fit to the data is shown. Different colors of the data points belong to different $\Omega$ values. Dots represent lines which are identified within $0.1 \AA$, and diamonds lines which differ more than $0.1 \AA$ from the theoretical position. The dotted lines represent the three $\sigma$ scatter around the fit. 

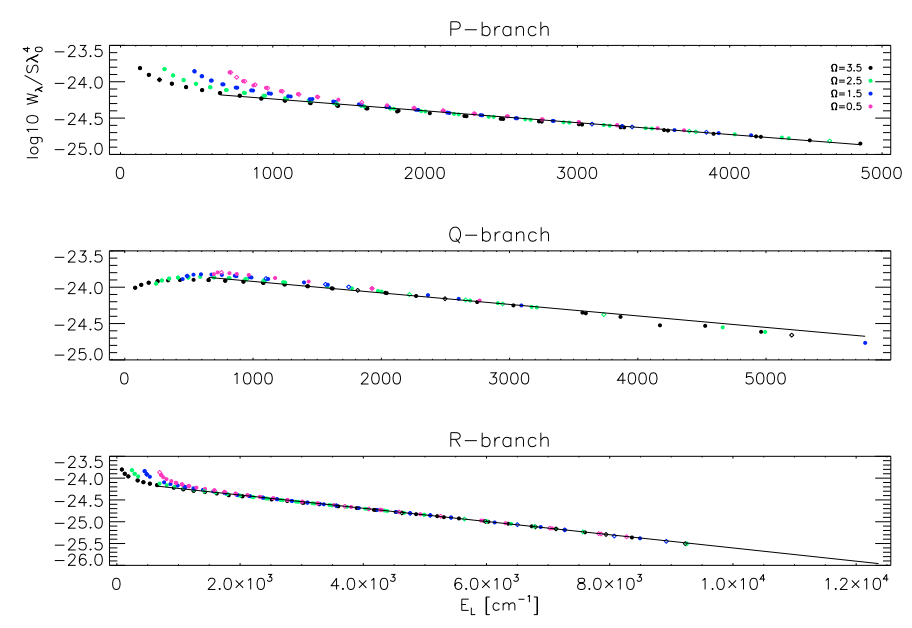

Fig. 10. Logarithm of the computed equivalent width and theoretical line strength against lower level energies for the $(0,0)$ band. In each plot a linear fit to the data is shown. Different colors of the data points belong to different $\Omega$ values. Dots represent lines which are identified within $0.1 \AA$, and diamonds lines which differ more than $0.1 \AA$ from the theoretical position.

identified vibrational bands, branches, and $\Omega$, with an appropriate estimated error (see Fig. 9).

In almost all cases a linear correlation is visible. In some cases where lines with small $J$ are present, we see a deviation from the straight line. This deviation is expected because of the different formation heights for the $\mathrm{FeH}$ lines. We calculated model plots with synthetic lines, which reproduce this behavior in detail (Fig. 10). The computations are very similar to the observations, which supports the assumption of different heights of formation. To test this assumption, we computed the contribution function for the FeH lines (Magain 1986) and used them to determine a weighted mean of the continuum optical depth, $\tau_{\text {mean }}$. The latter describes a representative atmospheric line formation depth. In Fig. 11, we plot $W_{\lambda}$ and $\tau_{\text {mean }}$ against $E_{0}$ for each branch from the $(0,0)$ band. These plots show clearly that the $\mathrm{P}$ - and R-lines with low and very high $E_{0}$ originate in deeper layers than the lines with medium $E_{0}$. We also see that the lines with low and high $E_{0}$ are the ones with small $W_{\lambda}$, because the Hönl-London factors for P- and R-lines are proportional to $J_{1}$ and hence the lines become stronger for high $J_{1}$, but they also decrease for high $J_{1}$ due to their increasing $E_{0} \mathrm{~s}$. Hence, there is a maximum in $W_{\lambda}$ for medium $J_{1}$. For Q-branch lines the situation is different. The Hönl-London factors decrease with increasing $J_{1}$ and hence the line strength monotonically decreases with $J_{1}$. In this case, the lines with low $E_{0}$ are the lines with the largest $W_{\lambda}$, and accordingly these lines are formed in higher layers and the Q-branch shows the opposite behavior to the P-and R-branches in the $\log _{10} \frac{W_{\lambda}}{S \lambda_{0}^{4}}$ plot.

We can conclude that in deeper layers, where weak lines are formed, the equivalent width is enhanced by the larger number of $\mathrm{FeH}$ molecules that contribute to the absorption (see Fig. 12). The molecule number increases towards deeper layers because of the higher overall density even though the concentration of $\mathrm{FeH}$ molecules relative to $\mathrm{Fe}$ and $\mathrm{H}$ decreases. Towards higher temperatures in deeper layers or in hotter stars, the number of $\mathrm{FeH}$ molecules would decrease again due to the ionisation of $\mathrm{Fe}$, which is then no longer available for the formation of the molecule. In our case, this behavior results in a deviation from a straight line in Fig. 9

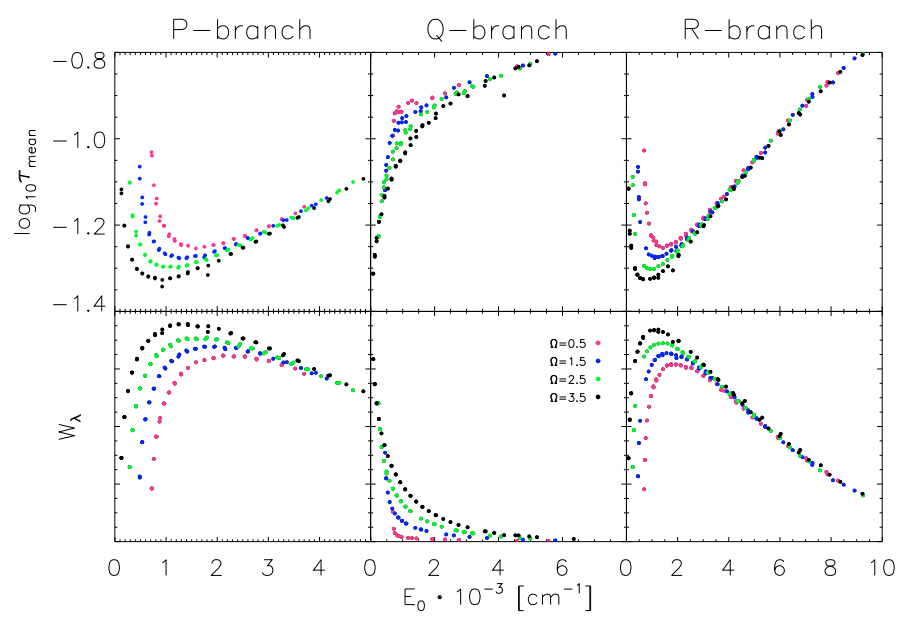

Fig. 11. $\log _{10} \tau_{\text {mean }}$ and $W_{\lambda}$ are plotted as a function of lower state energy for computed $\mathrm{FeH}$ lines from the $(0,0)$ band for the three branches.

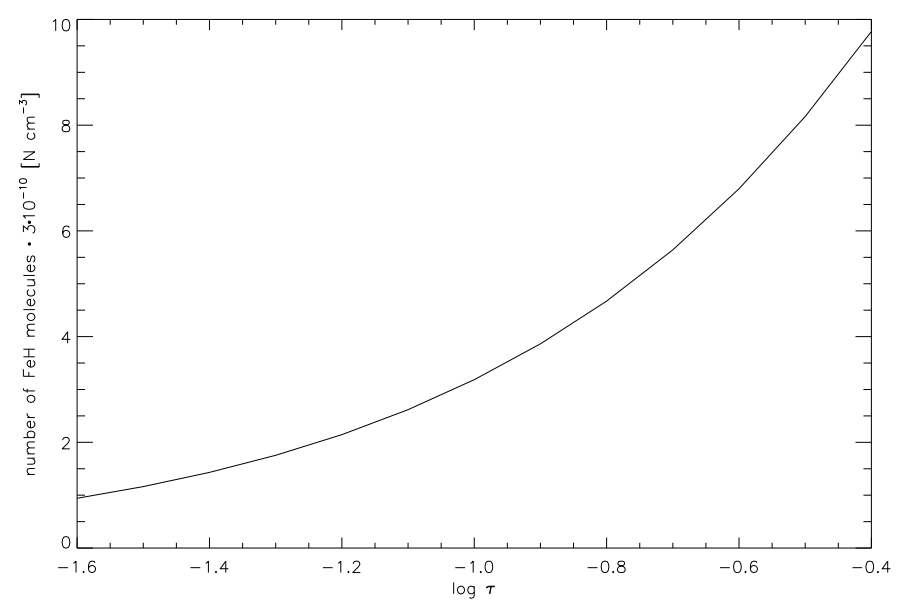

Fig. 12. The number of FeH molecules as a function of optical depth in the region where the $\mathrm{FeH}$ absorption lines are formed.

With this knowledge, we can use the line strength to confirm the identification of FeH. In Fig. 9, clearest correlations are found for the $(0,0)$ and $(1,1)$ bands, which also have the largest number of lines, but also for the P-branch of the $(1,0)$ and $(2,1)$ transitions and the R-branch of the $(2,2)$ transition. The $(3,2)$ and $(4,3)$ transitions also show linear correlations but with a larger scatter. This result is a strong indication that all the identified bands are present in the observed spectra. Although the Q-branches of the $(0,0)$ and $(1,1)$ bands show no clear linear dependence for large $E_{0}$, we expect them to be present and correctly identified, because they show the expected downward trend for low energies. The scatter towards higher energies is much larger than in the other branches, and there are only a few measurements. The slope seems to be positive, but we could expect it to be negative if we would have more data points.

We fitted the data points linearly for lines with $J_{1}>7$ (to avoid the region where the influence of variable $\mathrm{FeH}$ number is too strong) and used the difference of the data points from the fit as a measure for the confidence of an identified line. A line is classified as "P" if the difference between $\log _{10} \frac{W_{\lambda}}{S \lambda_{0}^{4}}$ and the linear fit is smaller than three times the standard deviation $\sigma$ of the scatter around the fit. But we assume that lines with $J \leq 7$ are also present, because they exactly show the expected behavior. It is classified as " $\mathrm{Pb}$ " if the deviation is greater than $\sigma$ and the data point is above the linear fit, and as " $\mathrm{R}$ " if it is below. It is 
Table 4. FeH molecular data of the identified lines.

\begin{tabular}{|c|c|c|c|c|c|c|c|c|c|c|c|c|c|c|c|}
\hline$\lambda_{\mathrm{obs}}$ & $\lambda_{\text {theo }}$ & $v_{1}$ & $v_{\mathrm{u}}$ & $\Omega_{1}$ & $\Omega_{\mathrm{u}}$ & $J_{1}$ & $J_{\mathrm{u}}$ & $B$ & $A$ & $s_{A}$ & $E_{1}$ & $\Delta \lambda$ & blend & class & comment \\
\hline 9900.4846 & 9900.4902 & 0 & 0 & 3.5 & 3.5 & 17.5 & 18.5 & 3 & 470924.44 & 0.8309 & 0.225 & 0.0056 & 1.000 & $\mathrm{P}$ & \\
\hline 9901.1049 & 9901.1175 & 0 & 0 & 3.5 & 3.5 & 14.5 & 15.5 & 3 & 462792.47 & 1.0695 & 0.154 & 0.0126 & 1.000 & $\mathrm{P}$ & \\
\hline 9901.4704 & 9901.4116 & 0 & 1 & 3.5 & 3.5 & 32.5 & 31.5 & 1 & 133973.65 & 0.3378 & 0.794 & -0.0588 & 1.000 & $P$ & \\
\hline 9903.9631 & 9903.9809 & 0 & 0 & 3.5 & 3.5 & 18.5 & 19.5 & 3 & 472886.88 & 1.1484 & 0.252 & 0.0178 & 0.998 & $\mathrm{P}$ & \\
\hline 9904.9843 & 9904.9913 & 0 & 0 & 3.5 & 3.5 & 13.5 & 14.5 & 3 & 458947.25 & 1.4267 & 0.134 & 0.0070 & 1.000 & $\mathrm{P}$ & \\
\hline 9905.8556 & 9905.8940 & 0 & 0 & 3.5 & 3.5 & 15.5 & 16.5 & 3 & 466010.13 & 1.1121 & 0.177 & 0.0384 & 0.969 & $\mathrm{P}$ & \\
\hline 9905.8556 & 9905.8940 & 3 & 4 & 3.5 & 3.5 & 5.5 & 4.5 & 1 & 93477.74 & 1.1121 & 0.649 & 0.0384 & 0.026 & $\mathrm{P}$ & \\
\hline$\vdots$ & $\vdots$ & $\vdots$ & $\vdots$ & $\vdots$ & $\vdots$ & $\vdots$ & $\vdots$ & $\vdots$ & $\vdots$ & $\vdots$ & $\vdots$ & $\vdots$ & : & : & : \\
\hline
\end{tabular}

Notes. The columns are described in more detail in the Appendix.

classified as "Q" if the line could not be investigated because, we only investigated lines with $\Delta \Omega=0$. We give a list of all identified lines with quantum numbers and corrected wavelength in Table $4^{4}$. This list is explained in more detail in the Appendix.

\subsection{Corrections to the line strengths}

If we wish to use Eq. (17) to correct for the differences in line depth, we have to match the stellar parameters as closely as possible. These parameters are basically effective temperature, surface gravity, and chemical composition, as well as van der Waals-broadening constants, whose influence becomes significant at these low temperatures. The van der Waals broadening was computed with Unsöld's hydrogenic approximation, and an enhancement factor was used to model the line wings correctly. Because no enhancement factor is reported for $\mathrm{FeH}$, we needed to determine one. For M-type stars, an assumed surface gravity of $\log g=5.0$ is standard and the chemical composition is usually assumed as solar. To match the strong Ti lines in the 10 300-10700 $\AA$ region as well as the FeH lines, we increased the iron abundance from 7.41 to the Grevesse \& Anders (1989) value of 7.63. We used this scaling as a parameter and do not claim this to be the actual iron abundance of GJ1002. The free parameters for GJ1002 are now $T_{\text {eff }}$, the van der Waalsbroadening enhancement factor (which we call from now on $\beta_{v \mathrm{~d} W}$ ), and the instrumental resolving power, which we used as a fitting parameter to account for possible additional rotational broadening. These three parameters are strongly correlated. We created $\chi^{2}$ maps to determine the most likely combination that best matches the observed spectra.

\subsection{1. $\chi^{2}$ maps}

For the comparison between observation and computation we chose a region where the original line list fits best, the lines are strongest and consequently the influence of van der Waals broadening is largest. We selected the first $100 \AA$ from the $(0,0)$ band head at $9900 \AA$. For the computations we used our new line list with corrected positions and also included the identified atomic lines. To create the $\chi^{2}$ maps for the three combinations of parameters, we searched for the minimum for each parameter (light cross in left plots in Fig. 13) and used this value to construct the $\chi^{2}$ map for the other two parameters. The $\chi^{2}$ maps (right hand

\footnotetext{
4 Full Table 4 is only available at the CDS.
}
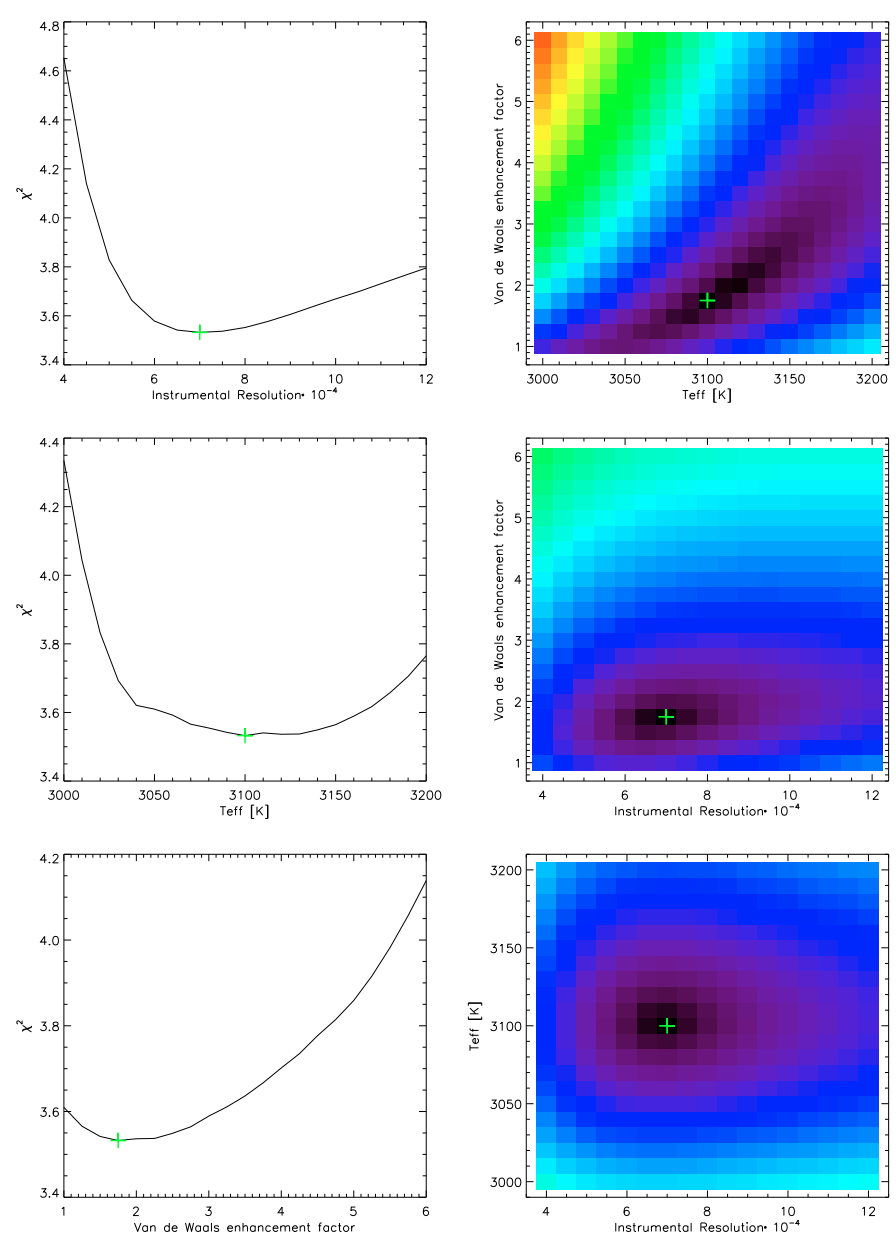

Fig. 13. Left: minima in $\chi^{2}$ plots for the resolving power, $T_{\text {eff }}$, and $\beta_{v \mathrm{~d} W}$ (from top to bottom). Right: $\chi^{2}$ maps for the three parameter combinations, $\beta_{v \mathrm{~d} W}-T_{\text {eff }}, \beta_{v \mathrm{~d} W}-$ resolving power, and resolving power $-T_{\text {eff }}$ (from top to bottom). The $\chi^{2}$ maps are computed for the minimum value of the leftover parameter on the left hand side.

side in Fig. 13) yield a consistent picture of the parameter combinations for the spectra of GJ1002.

The most likely parameters for effective temperature, resolving power, and $\beta_{v \mathrm{~d} W}$ are $3100 \mathrm{~K}, 70000$ and 1.75 , respectively. It is obvious, however, that the $\chi^{2}$ curves show regions with broad minima which would allow for variations in the derived parameters. 


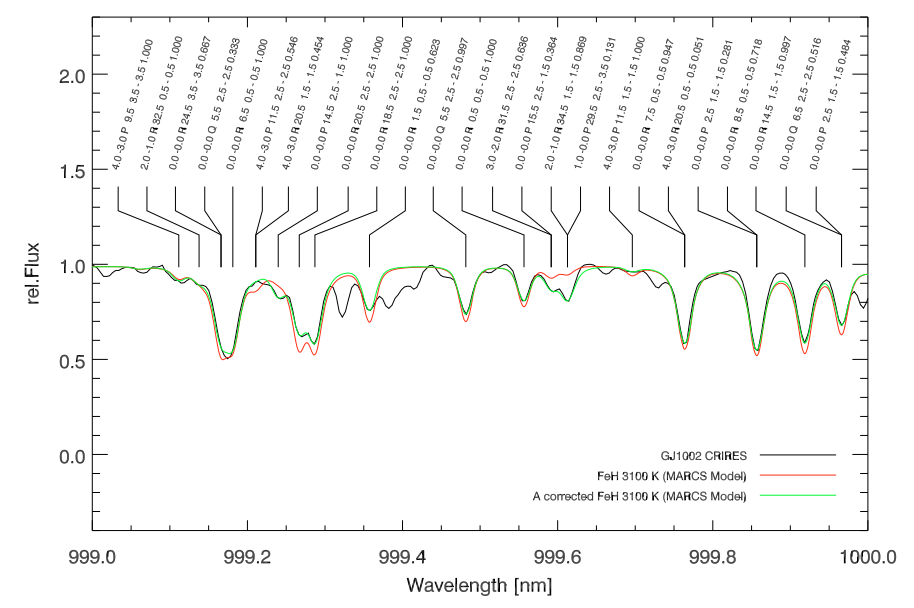

Fig. 14. Observed spectrum of GJ1002 (black) and computed one before A correction (red) and after A correction (green) labeled with quantum numbers (both with corrected positions).

Because the observed spectra are supposed to have a resolving power of 100000 , the difference to our determined resolving power stems probably from rotation, which is measured to be lower then $3 \mathrm{~km} \mathrm{~s}^{-1}$, but not necessarily zero. The difference in resolving power results in $v \sin i \approx 1.3 \mathrm{~km} \mathrm{~s}^{-1}$ at a wavelength of $10000 \AA$.

An independent constraint for the instrumental resolving power and effective temperature is given by the Ti lines, which are strong and distributed over a wide wavelength range in the spectra. The computation of these lines with the parameters and broadening constants given by VALD fits the observations within $5 \%$ for the line depth. This gives us confidence that the decreasing line depth of the $\mathrm{FeH}$ lines with increasing wavelength is a real feature and not due to normalization effects.

\subsubsection{Einstein $A$ values}

To correct for the Einstein A values we used computed spectra, which already have corrected line positions. We iteratively adjusted the Einstein A values because for saturated lines the first scaling is not sufficient. The scaling factors for each line are listed in Table 4, and an example for the corrected computed spectra is shown in Fig. 14. To estimate an error, we assumed an accuracy of $1 \%$ for the observed line depth, which results in an error of $\sim 3 \%$ for the scaling factors. If we furthermore assume that the line depth is modified by an unknown blended feature by, e.g., $5 \%$, an error of $\sim 16 \%$ follows. The accuracy of the Einstein A scaling is also influenced by the van der Waals-enhancement factor $\beta_{v \mathrm{~d} W}$. A change of \pm 1 gives a mean difference of $\sim 5 \%$ in the scaling constants, but can be up to $30 \%$ for individual lines, due to the logarithmic ratio of the intensities (see e.g. Eq. (17)).

We obtained a good fit to the data with the scaled Einstein A values even for some lines that were calculated to be very weak. Yet this results in some cases in unrealistic $\log g f$ values, and we assume that these weak lines are blended with unknown components. The scaling of line blends is a difficult problem because it results in equal scaling factors for lines with completely different quantum numbers. To avoid this problem one could determine scaling factors for each branch, but we chose the simpler scheme of scaling each line. We use Eq. (17) and plot $s$ for the Einstein A values against $J_{1}$ to search for a possible rotational dependence (Fig. 15). We plot only scaling factors for the $(0,0)$ and $(1,1)$ bands, because these are the bands with the largest

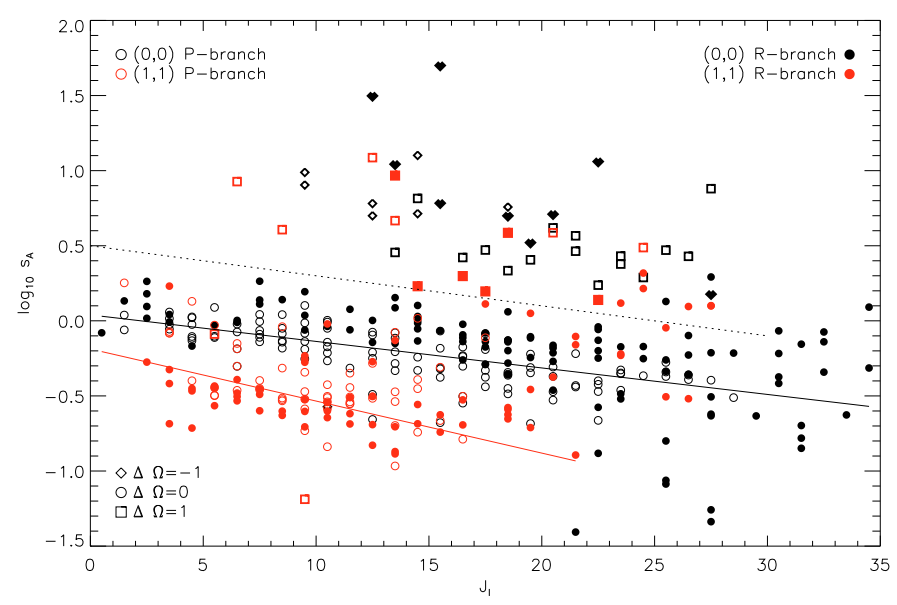

Fig. 15. Scaling factor for the Einstein A values against rotational quantum number for the $(0,0)$ and $(1,1)$ bands. The plot is truncated at $J_{1}=35$ for better visibility.

number of lines, and we used only lines which contribute more than $99 \%$ to a blended line feature to avoid contributions from incorrectly scaled lines.

We found two groups of scaling factors. One group describes a strong enhancement of the line depths ("positive" scaling factors) and the other only a small enhancement for low $J_{1}$ and a reduction towards lines with high $J_{1}$ ("negative" scaling factors). We divide these two groups by a dashed line in Fig. 15. For the $\Delta \Omega=0$ transitions of the $(0,0)$ and $(1,1)$ bands we include linear fits to the data in Fig. 15 to indicate the slope.

The group of positive scaling factors is strongly dominated by $\Delta \Omega \pm 1$ transitions (diamonds and rectangles), while the group of negative scaling factors consists of only $\Delta \Omega=0$ transitions (circles). The latter scaling factors for $(0,0)$ and $(1,1)$ bands (black and red circles, open for P-branches and filled for Rbranches) show an almost linear behavior with $J_{1}$ and become stronger towards higher $J_{1}$. The positive scaling factors also show two linear groups, which originate from the $(1,1)$ band (red rectangles) and from the $(0,0)$ band (black diamonds and rectangles). These scaling factors describe a strong enhancement of the lines for low $J_{1}$ and become smaller towards high $J_{1}$. All groups of scaling factors have a similar negative slope, and are only shifted by a constant factor to higher or lower scaling factors.

The distribution of other bands and lines that contribute less than $99 \%$ to a blended feature gives only a larger scatter to the data points, but does not change the basic trend of the scaling factors. We conclude that the $J_{1}$ dependence in the scaling factors likely indicates shortcomings in the calculated Hönl-London factors. In particular, satellite branches with $\Delta \Omega \pm 1$ are much stronger than expected for Hund's case (a). In other words, the two ${ }^{4} \Delta$ electronic states are heavily mixed with other electronic states and the simple Hund's case (a) behavior anticipated for isolated electronic states with relatively large spin-orbit splittings is not found.

\subsection{Rotational temperatures}

A rotational temperature $T_{\text {rot }}$ can be obtained from the slope $m$ of the linear fit in the $\left[\log _{10} W_{\lambda} / S, E_{0}\right]$ diagram described in the above section. $T_{\text {rot }}$ can be calculated from

$T_{\text {rot }}=\frac{h c}{m k} \log _{10} e$. 


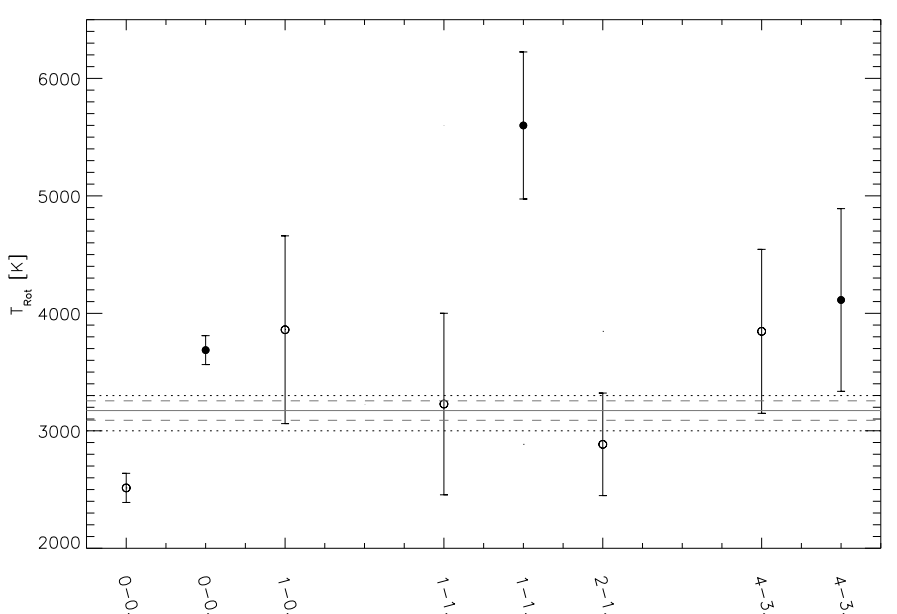

Fig. 16. Rotational temperatures derived from the slope of the linear fits in Fig. 9. The error bars indicate the one sigma level. The dotted lines give the expected upper and lower effective temperatures for a 5.5 M-dwarf. The grey solid line is the weighted mean of the rotational temperatures with its one sigma error (dashed-line).

For the fit to the data, we used only lines with $J_{1}>7$ to avoid the significant influence from a varying absorber number, and neglected lines with a line depth greater than 0.5 to avoid saturation effects.

Owing to the large errors (derived from the uncertainty of the slope of the linear fit in Fig. 9), we considered only rotational temperatures with moderately small one sigma errors (see Fig. 16).

We found systematically lower temperatures for the Pbranches in comparison to the R-branches, which is consistent with different heights of formation for most of the lines in a branch as seen in Fig. 11.

If we compute the weighted mean of the rotational temperatures (grey solid line in Fig. 16) with the $1 \sigma$-error (grey dashedline in Fig. 16), we obtain $\bar{T}_{\text {rot }} \approx 3200 \pm 100 \mathrm{~K}$. This is in the middle of the expected temperature range for this spectral type (3000 K-3300 K, dashed-dotted lines in Fig. 16) and is close to our estimated value of $\sim 3100 \mathrm{~K}$. The main contribution to $\bar{T}_{\text {rot }}$ stems from the P- and R-branches of the $(0,0)$ transition due to their small uncertainties. The weighted mean of the P- and R-branches are $\bar{T}_{\text {rot }}^{P} \approx 2600 \pm 150 \mathrm{~K}$ and $\bar{T}_{\text {rot }}^{R} \approx 3750 \pm 150 \mathrm{~K}$, respectively.

We point out that even for a single branch in a band the obtained temperature is an average over the individual excitation temperatures for each line. The resulting temperature crucially depends on the selection of the lines that are used. If one uses lines with similar equivalent width and lower level energy, then one could obtain temperatures for certain regions in the atmosphere. For this method a large number of lines is required, though because otherwise the uncertainties become too large.

We conclude that in order to use the method of rotational temperatures, a large number of well measured lines are required to minimize the error in the slope. Finally, the rotational temperature can only be expected to match the effective temperature if the lines form in a region around optical depth unity.

\section{Summary and conclusion}

We investigated the $z$-band region of the M5.5 dwarf GJ1002 with high-resolution CRIRES spectra. This is the region where the $(0,0)$ vibrational band of $\mathrm{FeH}$ is present ("Wing-Ford band"). We were able to identify the $(0,0),(1,0),(1,1),(2,1),(2,2)$, $(3,2)$, and $(4,3)$ bands. For confirmation of the band assignment, we used the method of coincidence, cross-correlation techniques, and the line intensities.

For the identified lines, we applied empirical corrections to the theoretical line positions. The deviations between observed and computed positions are Gaussian-distributed around zero. For small deviations $(<0.025 \AA)$, this could be due to uncertainties in the wavelength calibration of the observed spectra and in other cases due to uncertainties in the molecular constants which were used to generate the line list. Note that these empirical wavelength adjustments reproduce the observed stellar spectrum, but the quantum number assignments are by no means assured.

The method of coincidence confirms the presence of the $(0,0)$ and $(1,1)$ bands, but for the other bands we needed crosscorrelation techniques to show their presence. Again the $(0,0)$ and $(1,1)$ bands show clear peaks in the cross-correlation function even with an uncorrected line list, but for the other bands it was necessary to use corrections to the line list to confirm them. We also used line intensity information by plotting the ratio $W_{\lambda} / S \lambda_{0}^{4}$ against $E_{0}$, which shows a linear behavior if the band is present in the spectra. With this method we could confirm the presence of all other bands, although not uniquely for the Q-branches.

From the slope of the line in the $\left[W_{\lambda} / S \lambda_{0}^{4}, E_{0}\right]$ plots, it was possible to derive excitation temperatures for rotational transitions, which could be identified with the effective temperature of the star if the lines are formed in the photosphere. We showed that this method is very uncertain because the error in the slope is high. The derived temperatures for the individual vibrational transitions range from $\sim 2500 \mathrm{~K}$ to $\sim 5500 \mathrm{~K}$ for GJ1002, but the weighted mean $\bar{T}_{\text {rot }} \approx 3200 \pm 100 \mathrm{~K}$ is very close to the expected temperature of an M 5.5 dwarf. However, the large error bars and differences between P- and R-branch temperatures suggests that this agreement may be more of a coincidence than a physical result.

Finally we corrected the line strength in the FeH line list by scaling the Einstein A values, as some of these lines show large discrepancies compared to the observations. For this purpose it was necessary to derive the instrumental broadening (which included the rotational broadening), effective temperature, and an enhancement factor for the van der Waals-broadening constants. The instrumental resolving power was derived to be $R=70000$, which is equivalent to a rotational broadening with $v \sin (i) \sim 1.3 \mathrm{~km} \mathrm{~s}^{-1}$ since the observed $R=100000$. We also derived an effective temperature of $T_{\mathrm{eff}}=3100 \mathrm{~K}$, and a van der Waals-enhancement constant of 1.75. The scaling factors of the Einstein A values show an almost linear dependence on $J_{1}$, which indicates that there is likely a problem in the calculation of the Hönl-London factors.

With the improved identification of $\mathrm{FeH}$ lines, it is now possible to characterize the $\mathrm{FeH}$ lines in the $z$-band region (e.g. magnetically sensitive and insensitive lines, temperature sensitivities of individual lines). Our improved line list will aid in the identification and simulation of $\mathrm{FeH}$ lines in spectra of cool stars.

\section{Appendix}

\section{Explanation of the $\mathrm{FeH}$ table}

Table 4 contains the observed wavelength $\lambda_{\mathrm{obs}}$, which is obtained with the Voigt fit, and the theoretical wavelength $\lambda_{\text {theo }}$ from the 
list of Dulick et al. (2003). The wavelengths are in vacuum. We also give the quantum numbers of the lines and the Einstein $\mathrm{A}$ values with their scaling factors $s_{A}$. The lower-level energy $E_{l}$ is given in $\mathrm{eV}$. The difference in position $\Delta \lambda=\lambda_{\text {theo }}-\lambda_{\mathrm{obs}}$ is also printed in the table. If the line is a blended line, then its contribution to the blend is given as the fraction normalized to one. If the line is not blended, its blend value is one. We then give the classification of the line as defined in Sect. 3.3. We add a comment if the line is blended by an atomic feature, or if the classification of the line did not agree with the scaling factor of the Einstein A values.

\section{Explanation of the $\mathrm{FeH}$ atlas}

We plotted the whole spectrum in bins of $1 \mathrm{~nm}$ from $990 \mathrm{~nm}$ to $1076.6 \mathrm{~nm}$. Shown are the observed spectrum of GJ1002 (black), the computed spectrum with corrected positions (red), the computed spectrum with corrected positions and scaled Einstein A values (green). We also labeled all lines with $W_{\lambda} \geq 2 \mathrm{~m} \AA$ with quantum numbers for the vibrational transition, the branch, the lower $J$, the upper and lower $\Omega$, and in the last position, their blend fraction. The blend fraction is unity if a line is not blended. We also labeled the position of atomic lines with the element name below the spectrum.

Acknowledgements. S.W. acknowledges the support from the DFG Research Training Group GrK - 1351 "Extrasolar Planets and their host stars". A.R. acknowledges research funding from the DFG under an Emmy Noether Fellowship (RE 1664/4- 1). A.S. acknowledges financial support DFG under grant RE 1664/4- 1 and from NSF under grant AST07-08074. Some support to PFB was provided by the NASA laboratory astrophysics program. We thank P. Hauschildt and D. Shulyak for useful discussions.

\section{References}

Afram, N., Berdyugina, S. V., Fluri, D. M., et al. 2007, A\&A, 473, L1

Afram, N., Berdyugina, S. V., Fluri, D. M., Solanki, S. K., \& Lagg, A. 2008, A\&A, 482, 387
Baschek, B., Holweger, H., \& Traving, G. 1966, Astronomische Abhandlung der Hamburger Sternwarte, 8, 26

Bernath, P. 2005, Spectra of Atoms and Molecules (Oxford Oxfordshire: Oxford University Press)

Cushing, M. C., Rayner, J. T., Davis, S. P., \& Vacca, W. D. 2003, ApJ, 582, 1066

Dulick, M., Bauschlicher, Jr., C. W., Burrows, A., et al. 2003, APJ, 594, 651

Fawzy, D. E. 1995, Master's Thesis, M. Sci.-Thesis, Cairo University

Fawzy, D. E., Youssef, N. H., \& Engvold, O. 1998, A\&AS, 129, 435

Gray, D. F. 2008, The Observation and Analysis of Stellar Photospheres, The Observation and Analysis of Stellar Photospheres, ed. D. F. Gray (Cambridge: Cambridge University Press)

Grevesse, N., \& Anders, E. 1989, in Cosmic Abundances of Matter, ed. C. J. Waddington, AIP Conf. Ser., 183, 1

Grevesse, N., Asplund, M., \& Sauval, A. J. 2007, Space Sci. Rev., 130, 105

Gustafsson, B., Edvardsson, B., Eriksson, K., et al. 2008, A\&A, 486, 951

Hargreaves, R., Hinkle, K. H., Bauschlicher, C. W., et al. 2010, AJ

Kochukhov, O. P. 2007, in Physics of Magnetic Stars, 109

Kupka, F., Piskunov, N., Ryabchikova, T. A., Stempels, H. C., \& Weiss, W. W. 1999, A\&AS, 138, 119

Magain, P. 1986, A\&A, 163, 135

Nordh, H. L., Lindgren, B., \& Wing, R. F. 1977, A\&A, 56, 1

Phillips, J. G., Davis, S. P., Lindgren, B., \& Balfour, W. J. 1987, ApJS, 65, 721

Piskunov, N. E., Kupka, F., Ryabchikova, T. A., Weiss, W. W., \& Jeffery, C. S. 1995, A\&AS, 112, 525

Reiners, A., \& Basri, G. 2006, ApJ, 644, 497

Reiners, A., \& Basri, G. 2007, ApJ, 656, 1121

Russell, H. N., \& Bowen, I. S. 1929, ApJ, 69, 196

Sauval, A. J., \& Tatum, J. B. 1984, ApJS, 56, 193

Schadee, A. 1964, Bull. Astron. Inst. Netherlands, 17, 311

Schiavon, R. P., Barbuy, B., \& Singh, P. D. 1997, ApJ, 484, 499

Schweitzer, A., Hauschildt, P. H., Allard, F., \& Basri, G. 1996, MNRAS, 283, 821

Wallace, L., Hinkle, K., \& Livingston, W. 1998, An atlas of the spectrum of the solar photosphere from 13500 to $28000 \mathrm{~cm}^{-1}$ (3570 to $7405 \mathrm{~A}$ ), ed. L., Wallace, K., Hinkle, \& W., Livingston

Wallace, L., Livingston, W. C., Bernath, P. F., \& Ram, R. S. (ed.) 1999, An atlas of the sunspot umbral spectrum in the red and infrared from 8900 to $15050 \mathrm{~cm}^{-1}$ (6642 to 11230 [angstroms]), revised

Wende, S., Reiners, A., \& Ludwig, H. 2009, A\&A, 508, 1429

Wing, R. F. 1972, in Les Spectres des Astres dans l'Infrarouge et les Microondes, 123

Wing, R. F., \& Ford, W. K. J. 1969, PASP, 81, 527

Wöhl, H. 1970, Sol. Phys., 15, 342 